\title{
Impact of an educational programme on Alzheimer's disease patients' quality of life: results of the randomized controlled trial THERAD
}

Hélène Villars ${ }^{1 *}$, Christelle Cantet ${ }^{2}$, Eva de Peretti ${ }^{1}$, Amelie Perrin $^{1}$, Maria Soto-martin ${ }^{1}$ and Virginie Gardette ${ }^{1}$

\begin{abstract}
Background: Although educational interventions are recommended in Alzheimer's disease (AD), studies assessing the impact of interventions such as "therapeutic patient education" are scarce. Indeed, the intrinsic nature of the disease is considered a barrier to patients' involvement in such approaches. We aimed to evaluate an intervention by using a "dyadic" approach (patient and caregiver) in both intervention and assessment.

Methods: THERAD is a monocentric, randomized, controlled trial assessing the effects of a 2-month educational programme in mild to moderately severe AD patients among 98 dyads (caregiver/patient) on caregiver-reported patient quality of life (QOL) at 2 months. Community-dwelling patients and their caregivers were recruited in ambulatory units of the French Toulouse University Hospital. Self-reported patient QOL, autonomy, behavioural and psychological symptoms and caregiver $\mathrm{QOL}$ and burden were collected at 2,6 and 12 months. Linear mixed models were used in modified intention-to-treat populations. We also performed sensitivity analysis.

Results: A total of 196 dyads were included, 98 in each group. The mean age of the patients was 82 years, $67.7 \%$ were women, diagnosed with AD (+/- cerebrovascular component) (mean MMSE $=17.6)$, and $56.9 \%$ lived with a partner. The mean age of the caregivers was 65.7 years, and $64.6 \%$ were women (52.3\% offspring/42.6\% spouses), with a moderate burden (mean Zarit score $=30.9$ ). The mean caregiver-reported patient $\mathrm{QOL}$ was lower than the self-reported QOL (28.61 vs. 33.96). We did not identify any significant difference in caregiver-reported patients' QOL $(p=0.297)$ at 2 months, but there was a significant difference in self-reported patients' QOL at 2 months ( $p=$ $0.0483)$ or 6 months $(p=0.0154)$. No significant difference was found for the secondary outcomes. The results were stable in the sensitivity analyses.

Conclusions: This randomized controlled trial assessing an educational intervention in 196 dyads (Alzheimer's disease affected patient/caregiver) highlights the need to better consider the patient's point of view, since only the self-reported QOL was improved. Additional studies using this dyadic approach are necessary in targeted subpopulations of caregivers (spouse vs. child, gender) and of patients (severity of cognitive impairment or behavioural disturbances)
\end{abstract}

\footnotetext{
*Correspondence: helenevillars@gmail.com

${ }^{1}$ Geriatric Department, Toulouse University Hospital, Hopital La Grave- Cité de la Santé Place Lange TSA 60 033, 31059 Toulouse Cedex 9, France

Full list of author information is available at the end of the article
} 
Trial registration: THERAD study NCT01796314. Registered on February 19, 2013.

Keywords: Alzheimer's disease, Quality of life, Educational intervention, Caregiver, Dyadic approach, Inclusive approach

\section{Introduction}

Alzheimer's disease (AD) patients' care and support of their family is a major issue in the health care systems of Western countries [1]. By affecting one's cognition, emotional processes and behaviour, $\mathrm{AD}$ modifies the nature of the relationship between the person and his or her caregiver, usually a relative, and consequently the role of each individual in the family and social sphere [2]. Even if this change is sometimes positive for the relationship, it can lead to what has been called a "burden", which, shouldered by informal caregivers, has been reported in the literature as causing poor physical and mental health (depression, cardiovascular disease, anxiety) [3].

Unfortunately, there is currently a lack of safe and sufficiently effective pharmacological treatment to alleviate $\mathrm{AD}$ symptoms and their consequences on family life, leading to nonpharmacological therapies being placed at the forefront of therapeutic strategies [4].

Among the variety of nonpharmacological interventions designed to meet the complex needs of this population, despite not always being tested in highquality trials, "psychoeducational approaches" have become increasingly popular over the last two decades [5]. Several types of psychoeducational strategies have been developed in $\mathrm{AD}$, mostly offered either to caregivers or patients but, only recently, to the dyad (patient/caregiver). They mainly belong to multicomponent interventions, including a formalized educational programme and/or psychological support and/or respite and/or pharmacological treatment [6, 7]. The literature has reported positive effects for these multicomponent interventions, including psychoeducational interventions on caregivers' outcomes, such as knowledge and feelings of competence [8], depression [6], physical and mental health [9], anxiety [10], well-being and quality of life [11] and burden [7, $12,13]$, as well as on the patients' behavioural and psychological symptoms of dementia (BPSD) [14], well-being [15] and admission to long-stay care [16]. Negative results were reported for the patients' mood or cognition [17] and autonomy [18].

However, no study has evaluated an educational programme in isolation. The two interventions with the most "educational" content (not purely due to the inclusion of caregiver support) are DAISY [19] and AIDMA [18] but did not show any efficacy on patient outcomes: cognition [19] autonomy [18], quality of life (QOL) or behaviour $[18,19]$, or on the caregivers' depression, burden or QOL despite improvements in the "sense of competence" of the caregiver [19].

The most effective model of "educational intervention", called therapeutic patient education (TPE), is a "tailored person-centred lasting component of patient management", recommended for use with 60 diseases by the World Health Organization (WHO) and shown to be effective in many chronic conditions [20]. TPE is recommended in AD routine care [21], but there is currently a need for additional evidence about the most relevant manner to implement it. TPE aims to develop disease awareness and skills for self-management behaviours through validated tools delivered by specifically trained health professionals. TPE can induce selfmanagement behaviours and changes in patients' lifestyles in many chronic conditions (e.g. self-monitoring of asthma [22], adherence to medication in HIV/AIDS [23], health behaviours in general in cardiac rehabilitation [24]). However, in $\mathrm{AD}$, the intrinsic characteristics of the disease (cognitive impairment and anosognosia) have long been considered a real barrier to TPE implementation by $\mathrm{AD}$ patients themselves. Indeed, the patient's ability to acquire new skills has been assumed to be so impaired that it constitutes an obstacle to his or her involvement. For this reason, the caregiver, who bears a substantial burden, appeared first as the "real beneficiary" of any TPE approach in AD and the targeted population of TPE programmes in trials [25]. Nevertheless, psychoeducational approaches targeting caregivers (or both patients and caregivers) can potentially modify the caregiver's attitude, as demonstrated in paediatric care [26] and psychiatric care [27] to the benefit of the patient [27]. Thus, in light of these data, we assumed that TPE could induce a change in the caregiver's attitudes towards the $\mathrm{AD}$ patient, secondarily leading to a positive impact on the patient's quality of life. We therefore designed a trial assessing the impact of a TPE programme on AD patients' QOL from a "dyadic" perspective (caregiver/patient) [28] in both intervention and assessment by considering the "dyad" as the true beneficiary.

\section{Methods}

\section{Study design}

THERAD (Therapeutic Education in Alzheimer's Disease NCT01796314 in clinicaltrials.gov) is a monocentric, randomized, single blind, controlled trial assessing TPE in AD. Investigators and raters were 
blinded to group allocation. Details of the study protocol have been published previously [29].

\section{Participants}

In total, 196 dyads (patient/caregiver) were recruited. Patients were community-dwelling $\mathrm{AD}$ patients of all ages suffering from mild to moderately severe AD (MiniMental State Examination (MMSE) [30] score 11-26), with or without a cerebrovascular component, receiving support from a family caregiver (nonprofessional family member living with the patient or providing support at least 3 times a week or $8 \mathrm{~h}$ a week) were eligible for inclusion. The AD diagnosis was based on DSM-IV criteria, imaging (magnetic resonance imaging or computed tomography scan) and biology. Recruitment was performed in the memory clinic and geriatric units of the Toulouse University Hospital (TUH) between 1 January 2013 and 31 December 2015.

We conducted a sample size estimation based on the existing literature [31,32] and a pilot study we previously carried out [33]. The size was initially 170 dyads; however, during data monitoring, an unexpected $11 \%$ of unexploitable data for the primary outcome led us to include 26 additional dyads. This methodological choice appeared to be important for maintaining the statistical power of the results.

\section{Intervention}

The intervention consisted of a TPE programme of 2 months duration, as described in Fig. 1.

The intervention involved two individual sessions for patients and two for caregivers: at baseline (M0) and after 2 months (M2)). Additionally, the caregivers received four weekly group sessions (S1- S4) between M0 and M2. Individual and group sessions were conducted in the geriatric department of the TUH in a dedicated room of the ambulatory unit.

In the intervention group, each member of the dyad underwent a baseline "educational diagnosis", the first step of TPE. The patient was questioned by semidirective interviews on his or her representations and beliefs about $\mathrm{AD}$, life history, needs and requests. In a more open interview, the caregiver was questioned about his or her feelings and concerns, which helped in formulating a meaningful project for the dyad involving reachable goals and identifying skills to be acquired or strengthened. The individual session for the caregiver was 45 min, while the patient's session was more variable between individuals (from 15 to $45 \mathrm{~min}$ ).

The four weekly group sessions for caregivers were $3 \mathrm{~h}$ long and performed in small groups of six caregivers by multidisciplinary trained health professionals. Each session aimed to develop the caregivers' understanding of their relatives' illness (knowledge about the disease, crisis

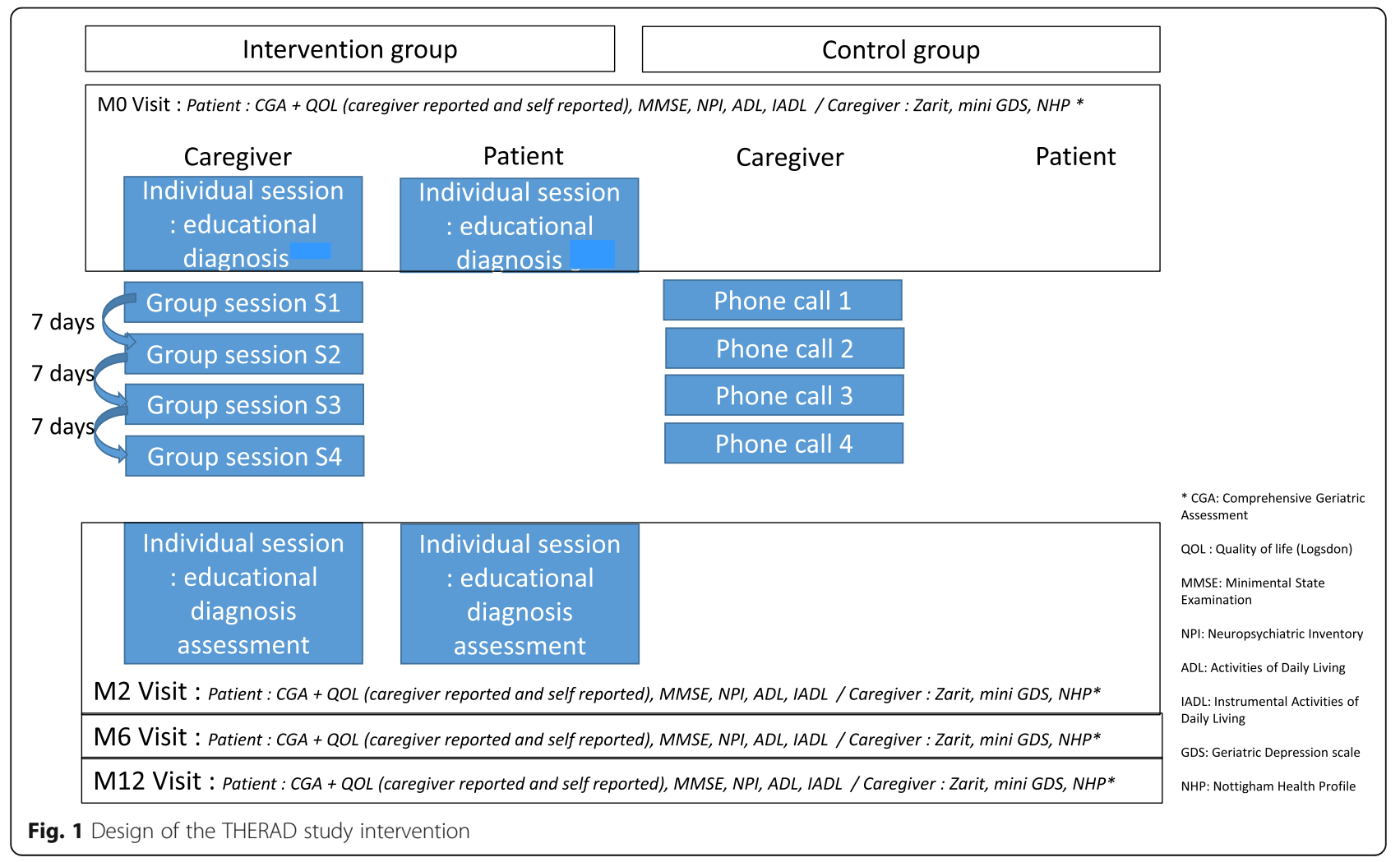


management of distressing or disruptive BPSD) and coping strategies (e.g. to adapt the communication style in stressful situations, strategies to find resources and a general understanding of care pathways) [29, 33]. The content used pedagogic methods and tools (storytelling, brainstorming, drawings, videos, quizzes) [34] designed to be reproducible. Each collective session was provided by the same professionals: a geriatrician and a nurse (S1 and S2) (a pharmacist also designed S2), a nurse and psychologist (S3) and a nurse and social worker (S4). The focus for the patient was the increase in their wellbeing in the daily caregiving relationship.

Last, just before the M2 visit, each member of the dyad benefitted from their second individual session to reformulate their objectives and classify them as "achieved" or "to reach". Additional advice was delivered, and satisfaction was collected from a questionnaire completed by both patients and caregivers. No joint session had been implemented.

The control group was designed as an "attention control group" (participants receiving social attention as subjects in the intervention group-but no other elements of the intervention). Indeed, participants benefited from routine medical care, and caregivers received phone calls of a short duration ( $5 \mathrm{~min}$ ) as a control condition, comprised of nonspecific and open-ended questions ("Did any change in your situation happen?" "How are you? How is the condition of your relative?") They were delivered weekly to have the same frequency of interactions with our team as subjects in the intervention group.

\section{Outcomes}

The primary outcome measure was a change in the $\mathrm{AD}$ patient's QOL at 2 months, on the Logsdon QOL-AD scale and rated by the primary caregiver [35]. The Logsdon QOL-AD is a 13-item questionnaire that uses a 4point Likert scale, with scores in the range $0-52$ points. This scale has been validated both for self-reporting (if MMSE scores $\geq 11$ ) and proxy (caregiver) reporting. We also collected the self-assessed Logsdon QOL-AD scores as a secondary outcome at 2, 6 and 12 months.

Several secondary endpoints were assessed at 2, 6 and 12 months: patient's BPSD based on the Neuro Psychiatric Inventory (NPI) [36] and functional autonomy based on the Activities of Daily Living scale (ADL) [37] and the Instrumental Activities of Daily Living scale (IADL) [38]; caregiver's burden through the Zarit Burden Inventory (ZBI) [39] and QOL based on the Nottingham Health Profile (NHP) [40].

We also collected several variables known to impact the patient's QOL [41]: caregiver's mood based on the mini Geriatric Depression Scale (GDS) [42] and patients' MMSE score [30]. Finally, patient and caregiver satisfaction data were collected from a questionnaire completed at 2 months.

\section{Statistical analysis}

Baseline characteristics of the participants who were included in the intention-to-treat (ITT) population (i.e. including all randomly assigned participants) are presented as the mean and standard deviation (SD) for quantitative variables and as frequency and percentage for qualitative variables. For some scales (Logsdon QOL-AD, MMSE, $\mathrm{ZBI}$ ), rare missing items were imputed up to $10 \%$ of the total number of items, or, as proposed by Logsdon [35] in the case of one or two missing items for the Logsdon QOL-AD scale; otherwise, the score was considered missing. The main imputation method used was the mean score of the remaining items, except for the NHP for which the proportionality rule was applied.

For continuous outcomes, linear mixed models, adjusted by the baseline data to take into account the regression to the mean [43], were used to assess the effect of the intervention (the mixed procedure from SAS). Analyses were performed on a modified ITT (mITT) population (i.e. including all randomly assigned participants with outcomes measured at baseline and with at least one post-baseline visit). For the binary outcomes, logistic mixed models in the ITT population, with the baseline value included in the dependent variable, were used (the Glimmix procedure from SAS).

For each mixed model, we included the following fixed effects: baseline value (only for continuous outcomes), intervention group, time as a continuous variable, and interaction between group and time. The mixed models included subject-specific random effects to take into account the intrasubject correlation: a random intercept to take into account the heterogeneity of the outcome at the first timepoint and a random slope (if significant) to take into account the heterogeneity of the slopes between subjects.

Subgroup analyses were performed to study the effect of the intervention according to the level of cognitive function (MMSE) and caregiver burden (ZBI) using linear mixed models as described above.

Two sensitivity analyses were performed for the Logsdon QOL score. The first analysis was conducted in the per-protocol population, excluding major protocol violations (poor compliance). A good TPE observance was defined by participation in 2 individual sessions and at least three or four collective sessions. The second one was performed with linear mixed models, including the baseline value in the dependent variable to include all randomized subjects and to model the trajectory with additional time. SAS version 9.4 (Cary, NC, USA) software was used for all analyses, and the statistical significance was set as $5 \%$. 


\section{Results}

A total of 196 dyads were included; 172 (87.8\%) were followed until 2 months, and 112 (57.2\%) completed the 12-month visit (Fig. 2). The baseline characteristics of the dyads are presented in Table 1 . With regard to the patients, their mean age was 82 years, $67.7 \%$ were women, $16.9 \%$ had a bachelor degree or higher, $56.9 \%$ lived with a partner, they were diagnosed with $\mathrm{AD}$ (or $\mathrm{AD}$ with a cerebrovascular component) for a mean time of 15 months, their mean MMSE was $17.65,48.2 \%$ had at least one incapacity of ADL, and their NPI mean score was 21.7 and $49.2 \%$ were being treated with antidementia drugs. The mean age of caregivers was 65.7 years, $64.6 \%$ were women, they were mainly a close relative (52.3\% were offspring, $42.6 \%$ were spouses), living at home with the patient (53.3\% spending a mean time of $21.67 \mathrm{~h}$ per week in caregiving) with a moderate burden (mean ZBI score $=30.9$ ) and a low QOL (mean NHP score $=119.60$ ). These characteristics were well balanced between the groups, with little heterogeneity in the patients' comorbidities, dementia aetiology, time elapsed since diagnosis, two NPI items (hallucinations, apathy) or speech therapy.

Regarding compliance, 87 patients (88.8\%) and 74 caregivers $(75.5 \%)$ showed good TPE observance.

The estimated changes in outcomes from baseline are presented in Table 2 and detailed below.

\section{Quality of life caregiver-reported (Fig. 3A)}

At 2 months, the change from baseline in the patient's QOL reported by the caregiver was 0.77 (95\% CI [0.13, 1.66]) for the intervention group and 0.09 (95\% CI [$0.83,-1.00])$ for the control group, representing a nonsignificant 0.68 -point difference $(95 \%$ CI $[-0.60,1.95] ; p$ $=0.297$ ) between groups.

The difference in QOL observed at 6 and 12 months decreased to 0.33 and -0.19 , respectively, but was not statistically significant.

\section{Quality of life self-reported by the patient (Fig. 3A')}

At 2 months, the self-reported patients' QOL increased by $0.72(95 \% \mathrm{CI}[-0.44,1.88])$ for the intervention group and decreased by -0.98 (95\% CI [- 2.19, 0.24]) for the control group, representing a significant 1.70 point difference $(95 \% \mathrm{CI}[0.01,3.38])$ in favour of TPE $(p=0.0483)$, which was sustained at 6 months (1.74, 95\% CI $[0.34,3.1] ; p=0.0154)$ but of borderline significance at 12 months $(p=0.0575)$.

No statistically significant difference was found for the other secondary outcomes.

\section{Sensitivity analyses}

The per protocol analyses produced stable conclusions (Table 3). The effect observed for self-reported QOL was enhanced (Fig. 3B, B').

\section{Subgroup analyses}

Table 4 presents the results of caregiver-reported QOL and self-reported QOL in the subgroup analysis.

We observed a significant effect of the intervention on the caregiver-reported QOL at 6 and 12 months in the subgroups of subjects with ZBI scores between $>40$ and $\leq 20(p=0.0129$ and $p=0.0163$, respectively) and at 2 months $(p=0.0390)$ in the subgroups with ZBI scores between 20 and 40 and $\leq 20$.

There was no difference in the self-reported patient QOL according to the level of burden (although we observed a significant positive effect of the intervention in the subgroup of subjects with ZBI score $>40$ at 2 months $(p=0.0069)$ and 6 months $(p=0.0026))$ or the MMSE scores (even though there was a significant positive effect of the intervention in the subgroup of the subjects with MMSE score $15-20$ at $2(p=0.0360)$ and 6 months $(p=0.0166))$.

Last, of the 73 caregivers who completed the satisfaction questionnaire at M2 (participation rate $=83.9 \%$ ), $26 \%$ were satisfied $(n=19)$ and $74 \%$ were very satisfied $(n=54)$.

\section{Discussion}

THERAD did not find any significant effect of TPE on the caregiver-reported patient's QOL at 2 months but there was a significant effect when it was self-reported by the patient. No other significant effect on either patient or caregiver outcomes was seen. Several reasons can explain this result. First, QOL is a multidimensional relevant criterion and a key patient-centred outcome $[41,44,45]$ especially in AD care [46], and when measuring the overall objectives of an educational intervention [47], measuring the QOL of persons with dementia is challenging because of the intrinsic nature of the disease: cognitive impairment, memory loss and anosognosia [48-50]. Indeed, the patient's ability to remember the past and thus to identify changes and make choices among items on a scale is affected by memory impairment but also by a lack of insight [48] and anosognosia $[43,44]$. These symptoms, which are more prevalent as the patient's condition worsens [50], tend to increase QOL scores and lead to a stability of QOL scores over time [44]. For this reason, the validity of self-reported QOL assessments by patients with dementia is a critical issue [51]. Therefore, researchers commonly use the rating by the caregiver as a proxy of the patient's QOL in most dementia clinical trials and this is considered reliable [52]. However, this proxy rating may introduce bias because of the influence of the caregiver's point of view and other factors that might influence their assessment of the patient's QOL $[47,53]$. In fact, discrepancies have been previously reported between self- and proxyreported QOL $[45,52,54]$, with caregivers 
437 Dyads assessed for eligibility

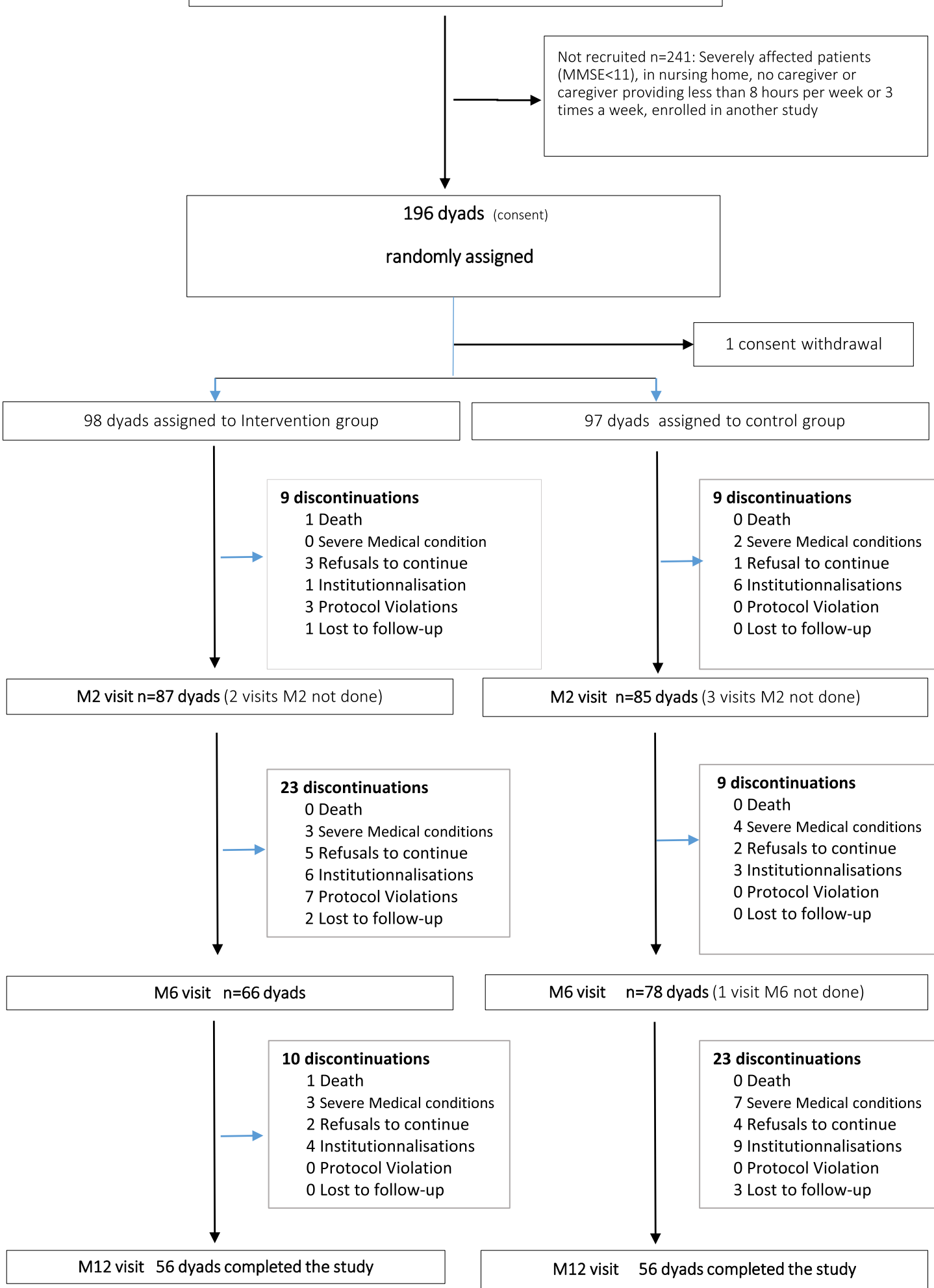

Fig. 2 Flowchart of the THERAD study population 
Table 1 Baseline dyads' characteristics

\begin{tabular}{|c|c|c|c|}
\hline \multirow[t]{2}{*}{ A. Patients' characteristics* } & \multirow{2}{*}{$\begin{array}{l}\text { Total population } \\
n=195 \\
\text { Mean (SD) or } n \\
(\%)\end{array}$} & \multicolumn{2}{|l|}{ Group } \\
\hline & & $\begin{array}{l}\text { Intervention } n=98 \\
\text { Mean (SD) or } n(\%)\end{array}$ & $\begin{array}{l}\text { Control } n=97 \\
\text { Mean (SD) or } n(\%)\end{array}$ \\
\hline \multicolumn{4}{|l|}{ Sociodemographic data } \\
\hline Age (years) & $82.03(5.88)$ & $81.94(6.38)$ & $82.12(5.36)$ \\
\hline $1:<75$ years & $22(11.28 \%)$ & $81.94(5,88)$ & $82.12(5.36)$ \\
\hline 2: $[75-85[$ years & $108(55.38 \%)$ & $13(13.27 \%)$ & $9(9.28 \%)$ \\
\hline 3: $\geq 85$ years & 65 (33.33\%) & $49(50.00 \%)$ & $59(60.82 \%)$ \\
\hline Women & $132(67.69 \%)$ & $66(67.35 \%)$ & $66(68.04 \%)$ \\
\hline \multicolumn{4}{|l|}{ Educational level } \\
\hline Elementary or no formal & $51(26.15 \%)$ & $20(20.41 \%)$ & $31(31.96 \%)$ \\
\hline Primary school certificate or less & $60(30.77 \%)$ & $36(36.73 \%)$ & $24(24.74 \%)$ \\
\hline Secondary education/high school & $51(26.15 \%)$ & $25(25.51 \%)$ & $26(26.80 \%)$ \\
\hline Bachelor's degree and higher & $33(16.92 \%)$ & $17(17.35 \%)$ & $16(16.49 \%)$ \\
\hline Professional activity in the past & $173(88.72 \%)$ & $88(89.80 \%)$ & $85(87.63 \%)$ \\
\hline Living in a marital status & $111(56.92 \%)$ & $56(57.14 \%)$ & $55(56.70 \%)$ \\
\hline Alzheimer's disease & $161(82.56 \%)$ & $87(88.78 \%)$ & $74(76.29 \%)$ \\
\hline Mixed dementia ( $A D$ and vascular) & $34(17.44 \%)$ & $11(11.22 \%)$ & $23(23.71 \%)$ \\
\hline MMSE mean (SD) & $17.65(4.11)$ & $18.12(4.04)$ & $17.16(4.14)$ \\
\hline \multicolumn{4}{|l|}{ Stage of severity (MMSE) } \\
\hline [21-26] & $51(26.15 \%)$ & $30(30.61 \%)$ & $21(21.65 \%)$ \\
\hline$[15 ; 20]$ & $77(39.49 \%)$ & $38(38.78 \%)$ & $39(40.21 \%)$ \\
\hline [11-15] & $67(34.36 \%)$ & $30(30.61 \%)$ & $37(38.14 \%)$ \\
\hline Time elapsed since diagnosis (months), $(n=176)$ & $14.57(19.25)$ & $12.44(18.97)$ & $16.80(19.39)$ \\
\hline Comorbidities CIRS-G & $9.91(3.85)$ & $9.30(3.82)$ & $10.53(3.80)$ \\
\hline \multicolumn{4}{|l|}{ Functional autonomy } \\
\hline ADL & $5.33(0.89)$ & $5.42(0.79)$ & $5.23(0.97)$ \\
\hline Score $<6 / 6$ (at least one incapacity) & $101(51.79 \%)$ & $46(46.94 \%)$ & $55(56.70 \%)$ \\
\hline IADL $(n=193)$ & $1.61(1.23)$ & $1.63(1.24)$ & $(1.22)$ \\
\hline Score 0-1 ( $\geq 4$ incapacities) & $102(52.85 \%)$ & $52(54.17 \%)$ & $50(51.55 \%)$ \\
\hline Gait and balance one leg balance $<5 \mathrm{~s}(n=184)$ & $121(65.76 \%)$ & $60(64.52 \%)$ & $61(67.03 \%)$ \\
\hline Visual impairment & $111(56.92 \%)$ & $56(57.14 \%)$ & $55(56.70 \%)$ \\
\hline Hearing impairment & $40(20.51 \%)$ & $17(17.35 \%)$ & $23(23.71 \%)$ \\
\hline Quality of life hetero-assessed by caregivers $(n=185)$ & $28.61(5.24)$ & $29.33(5.12)$ & $27.84(5.27)$ \\
\hline Quality of life self-assessed by patients $(n=145)$ & $33.93(6.03)$ & $33.66(5.53)$ & $34.24(6.59)$ \\
\hline \multicolumn{4}{|l|}{ Behavioural and psychological symptoms } \\
\hline NPI total score $(n=178)$ & $21.77(18.40)$ & $22.25(18.82)$ & $21.26(18.05)$ \\
\hline Delusions & $43(22.05 \%)$ & $17(17.35 \%)$ & $26(26.80 \%)$ \\
\hline Hallucinations $(n=194)$ & $38(19.59 \%)$ & $13(13.40 \%)$ & $25(25.77 \%)$ \\
\hline Agitation or aggressiveness ( $n=193$ ) & $105(54.40 \%)$ & $50(51.02 \%)$ & $55(57.89 \%)$ \\
\hline Depression/dysphoria $(n=194)$ & $116(59.79 \%)$ & $59(60.82 \%)$ & 57 (58.76\%) \\
\hline Anxiety $(n=194)$ & 140 (72.16\%) & 74 (75.51\%) & $66(68.75 \%)$ \\
\hline Euphoria & $41(21.03 \%)$ & $23(23.47 \%)$ & $18(18.56 \%)$ \\
\hline Apathy & 115 (58.97\%) & $51(52.04 \%)$ & $64(65.98 \%)$ \\
\hline Disinhibition, $(n=190)$ & $51(26.84 \%)$ & $20(20.83 \%)$ & $31(32.98 \%)$ \\
\hline
\end{tabular}


Table 1 Baseline dyads' characteristics (Continued)

\begin{tabular}{|c|c|c|c|}
\hline \multirow[t]{2}{*}{ A. Patients' characteristics* } & \multirow{2}{*}{$\begin{array}{l}\text { Total population } \\
n=195 \\
\text { Mean (SD) or } n \\
(\%)\end{array}$} & \multicolumn{2}{|l|}{ Group } \\
\hline & & $\begin{array}{l}\text { Intervention } n=98 \\
\text { Mean (SD) or } n(\%)\end{array}$ & $\begin{array}{l}\text { Control } n=97 \\
\text { Mean (SD) or } n(\%)\end{array}$ \\
\hline Irritability $(n=194)$ & $125(64.43 \%)$ & $61(62.24 \%)$ & $64(66.67 \%)$ \\
\hline Aberrant motor activity $(n=194)$ & $40(20.62 \%)$ & $21(21.43 \%)$ & $19(19.79 \%)$ \\
\hline Sleep disorders $(n=192)$ & $73(38.02 \%)$ & $35(36.08 \%)$ & $38(40.00 \%)$ \\
\hline Eating disorders $(n=192)$ & $76(39.58 \%)$ & $37(38.54 \%)$ & $39(40.63 \%)$ \\
\hline \multicolumn{4}{|l|}{ Pharmacological therapies } \\
\hline Acetylcholinesterase inhibitors or NMDA receptor blocker & $96(49.23 \%)$ & $43(43.88 \%)$ & $53(54.64 \%)$ \\
\hline Psychotropes & $46(23.59 \%)$ & $25(25.51 \%)$ & $21(21.65 \%)$ \\
\hline Antipsychotic drug & $5(2.56 \%)$ & $1(1.02 \%)$ & $4(4.12 \%)$ \\
\hline Anxiolytics & 19 (9.74\%) & $8(8.16 \%)$ & $11(11.34 \%)$ \\
\hline Sedative & $8(4.10 \%)$ & $7(7.14 \%)$ & $1(1.03 \%)$ \\
\hline Antidepressant therapy & $26(13.33 \%)$ & $14(14.29 \%)$ & $12(12.37 \%)$ \\
\hline \multicolumn{4}{|l|}{ Nonpharmacological therapies } \\
\hline Physical therapist & $27(13.85 \%)$ & $13(13.27 \%)$ & $14(14.43 \%)$ \\
\hline Ergotherapist & $4(2.05 \%)$ & $2(2.04 \%)$ & $2(2.06 \%)$ \\
\hline Psychologist & $1(0.51 \%)$ & $0(0.00 \%)$ & $1(1.03 \%)$ \\
\hline Speech therapist & $24(12.31 \%)$ & $19(19.39 \%)$ & $5(5.15 \%)$ \\
\hline Day care centre & $12(6.15 \%)$ & $6(6.12 \%)$ & $6(6.19 \%)$ \\
\hline Home help (daily living activities) & $38(19.49 \%)$ & $18(18.37 \%)$ & $20(20.62 \%)$ \\
\hline Domestic help (cleaning) & $23(11.79 \%)$ & $15(15.31 \%)$ & $8(8.25 \%)$ \\
\hline Nurse & $58(29.74 \%)$ & $26(26.53 \%)$ & $32(32.99 \%)$ \\
\hline Specialized nurse & $6(3.08 \%)$ & $3(3.06 \%)$ & $3(3.09 \%)$ \\
\hline Home meal deliveries & $2(1.03 \%)$ & $1(1.02 \%)$ & $1(1.03 \%)$ \\
\hline \multirow[t]{2}{*}{ B. Caregivers' characteristics ${ }^{a}$} & \multirow{2}{*}{$\begin{array}{l}\text { Total population } \\
n=195\end{array}$} & \multicolumn{2}{|l|}{ Group } \\
\hline & & $\begin{array}{l}\text { Intervention } \\
n=98\end{array}$ & $\begin{array}{l}\text { Control } \\
n=97\end{array}$ \\
\hline \multicolumn{4}{|l|}{ Sociodemographic data } \\
\hline Age (years) & $65.75(12.62)$ & $66.13(12.59)$ & $65.36(12.69)$ \\
\hline$\leq 65$ & $106(54.36 \%)$ & $49(50.00 \%)$ & $57(58.76 \%)$ \\
\hline$>65$ & $89(45.64 \%)$ & $49(50.00 \%)$ & $40(41.24 \%)$ \\
\hline Gender = women & $126(64.62 \%)$ & $60(61.22 \%)$ & $66(68.04 \%)$ \\
\hline \multicolumn{4}{|l|}{ Educational level, ( $n=192$ ) } \\
\hline Primary school certificate or less & $29(15.10 \%)$ & $14(14.43 \%)$ & $15(15.79 \%)$ \\
\hline Secondary education/high school & $60(31.25 \%)$ & $30(30.93 \%)$ & $30(31.58 \%)$ \\
\hline Bachelor's degree and higher & $103(53.65 \%)$ & $53(54.64 \%)$ & $50(52.63 \%)$ \\
\hline Professionally active (or in the past) $(n=191)$ & $179(93.72 \%)$ & $92(94.85 \%)$ & $87(92.55 \%)$ \\
\hline \multicolumn{4}{|l|}{ Caregiver status } \\
\hline Child & $102(52.31 \%)$ & $48(48.98 \%)$ & $54(55.67 \%)$ \\
\hline Spouse & $83(42.56 \%)$ & $44(44.90 \%)$ & $39(40.21 \%)$ \\
\hline Brother or sister & $1(0.51 \%)$ & $0(0.00 \%)$ & $1(1.03 \%)$ \\
\hline Nephew/niece & $3(1.54 \%)$ & $1(1.02 \%)$ & $2(2.06 \%)$ \\
\hline Daughter-in-law or son-in-law & $6(3.08 \%)$ & $5(5.10 \%)$ & $1(1.03 \%)$ \\
\hline Living in a marital status & $160(82.05 \%)$ & $85(86.73 \%)$ & 75 (77.32\%) \\
\hline
\end{tabular}


Table 1 Baseline dyads' characteristics (Continued)

\begin{tabular}{|c|c|c|c|}
\hline \multirow[t]{2}{*}{ A. Patients' characteristics* } & \multirow{2}{*}{$\begin{array}{l}\text { Total population } \\
n=195 \\
\text { Mean (SD) or } n \\
\text { (\%) }\end{array}$} & \multicolumn{2}{|l|}{ Group } \\
\hline & & $\begin{array}{l}\text { Intervention } n=98 \\
\text { Mean (SD) or } n(\%)\end{array}$ & $\begin{array}{l}\text { Control } n=97 \\
\text { Mean (SD) or } n(\%)\end{array}$ \\
\hline \multicolumn{4}{|l|}{ Living arrangement } \\
\hline Caregiver living at home with the patient & $104(53.33 \%)$ & $54(55.10 \%)$ & $50(51.55 \%)$ \\
\hline Patient and caregiver living apart & $91(46.67 \%)$ & $44(44.90 \%)$ & $47(48.45 \%)$ \\
\hline$<6.21$ miles & $53(27.18 \%)$ & $25(25.51 \%)$ & $28(28.87 \%)$ \\
\hline$>6.21$ miles & $38(19.49 \%)$ & $19(19.39 \%)$ & $19(19.59 \%)$ \\
\hline \multicolumn{4}{|l|}{ Length of caregiving ( $n=193$ ) years } \\
\hline $1:<1$ year & $53(27.46 \%)$ & $26(26.80 \%)$ & $27(28.13 \%)$ \\
\hline 2: between 1 and 3 years & $84(43.52 \%)$ & $42(43.30 \%)$ & $42(43.75 \%)$ \\
\hline 3: > 3 years & $56(29.02 \%)$ & $29(29.90 \%)$ & $27(28.13 \%)$ \\
\hline Hours of caregiving per week & $21.67(13.66)$ & $22.68(15.19)$ & $20.65(11.91)$ \\
\hline Medical chronic condition & $67(34.36 \%)$ & $38(38.78 \%)$ & $29(29.90 \%)$ \\
\hline \multicolumn{4}{|l|}{ Level of exhaustion and burden $(n=194)$} \\
\hline Zarit score $(n=194)$ mean & $30.89(15.77)$ & $29.97(16.19)$ & $31.83(15.36)$ \\
\hline $1:[0-20]$ & $58(29.90 \%)$ & $41(41.84 \%)$ & $40(41.67 \%)$ \\
\hline 2: [20-40] & $81(41.75 \%)$ & $24(24.49 \%)$ & $31(32.29 \%)$ \\
\hline $3:>40$ & $55(28.35 \%)$ & $55(56.70 \%)$ & $58(59.79 \%)$ \\
\hline Quality of life (NHP score) & $119.60(112.00)$ & $119.18(108.65)$ & $120.02(115.78)$ \\
\hline
\end{tabular}

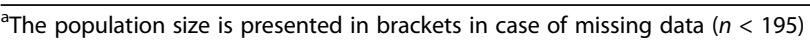

underestimating the patient's QOL $[45,55,52]$ especially in the cases when they are suffering from depression [52] or exhaustion [45] themselves, as well as depression [56] or BPSD [52] in their relative suffering from AD. However, acknowledging these discrepancies and the potential bias of proxy reporting, we made the choice to use proxy-reported QOL and to also consider selfreported QOL because it was demonstrated to be complementary $[52,53,57]$ and feasible by the QOL Logsdon scale. Indeed, scales were numerous [51], but the QOL-AD of Logsdon validated for both patient and caregiver use [35] was considered, and still is, as having valuable conceptual qualities [41, 51] This methodological choice led us to try to limit evaluation bias as much as possible by designing a real "attention control group" (previously described). We also created an intervention nonspecifically designed to reduce the burdenknown to influence proxy-rated QOL-but to preferentially improve knowledge and skills.

Furthermore, we thought this choice would also reflect our ethical position of a patient considered a "subject" of care rather than an "object" of care. Indeed, regarding secondary outcomes, our negative results are in accordance with those of the literature, with educational interventions found to have negative effects on the patients' cognition [19], autonomy [18] and mood [17]. We did not find any effect on the caregiver burden, anxiety or depression, whereas the literature generally reports positive effects $[6,7,10,12,22]$

Nevertheless, our results are balanced since while there was no improvement in the patient's QOL proxyrated, the self-rated QOL was significantly increased by TPE. We will first discuss the negative result of the QOL proxy-rated and then the positive results of the self-reported QOL.

Many reasons can be given to explain the negative result on proxy-rated QOL. First, regarding the characteristics of our population of $\mathrm{AD}$ patients, we observed that, as reported in the literature, patients assessed their QOL as higher than the assessment by their caregivers $[45,50,52$, 55], and characteristics known to influence caregiverreported QOL were found in THERAD (loss of functional independence [50], depression [56, 41] and apathy [52]). The level of BPSD was also relatively low in the patients (mean NPI 21), although this type of intervention is effective for BPSD [14], and BPSD is known to negatively impact (proxy or self-reported) QOL [52]. A sample of more severely affected patients (higher BPSD) may have been more pertinent to measure the impact. Our population sample in terms of the severity of the disease may also not be homogeneous enough. Indeed, the determinants of QOL in $\mathrm{AD}$ are different between stages; QOL is related to cognitive function during the mild stage and autonomy in moderate to moderately severe stages in the literature 
Table 2 Effect of the THERAD intervention vs control on primary and secondary outcomes: change from baseline to 2, 6 and 12 months (using linear mixed models in modified intention-to-treat population for continuous outcomes, and logistic mixed models in intention-to-treat population for binary outcomes)

\begin{tabular}{|c|c|c|c|c|c|c|c|c|c|}
\hline \multirow{3}{*}{ Outcome } & \multicolumn{2}{|c|}{$\begin{array}{l}\text { Estimated change } \\
\text { from baseline } \\
\text { mean }^{a} \text { or OR } \\
(95 \% \mathrm{Cl})\end{array}$} & \multirow{2}{*}{$\begin{array}{l}\text { Estimated } \\
\text { differences in } \\
\text { change from } \\
\text { baseline } \\
\text { mean or } \mathrm{OR}^{\mathrm{b}} \\
(95 \% \mathrm{Cl}) \\
p \text { value } \\
\text { Intervention vs } \\
\text { Control }\end{array}$} & \multicolumn{2}{|c|}{$\begin{array}{l}\text { Estimated change } \\
\text { from baseline } \\
\text { mean }^{\mathrm{a}} \text { or OR } \\
(95 \% \mathrm{Cl})\end{array}$} & \multirow{3}{*}{$\begin{array}{l}\text { Estimated } \\
\text { differences in } \\
\text { change from } \\
\text { baseline } \\
\text { mean or OR } \\
(95 \% \mathrm{Cl}) \\
p \text { value } \\
\text { Intervention vs } \\
\text { Control }\end{array}$} & \multicolumn{2}{|c|}{$\begin{array}{l}\text { Estimated change } \\
\text { from baseline } \\
\text { mean }^{\mathrm{a}} \text { or OR } \\
(95 \% \mathrm{Cl})\end{array}$} & \multirow{2}{*}{$\begin{array}{l}\text { Estimated } \\
\text { differences in } \\
\text { change from } \\
\text { baseline } \\
\text { mean or } O R^{b} \\
(95 \% \mathrm{Cl}) \\
p \text { value } \\
\text { Intervention vs } \\
\text { Control }\end{array}$} \\
\hline & Intervention & Control & & Intervention & Control & & Intervention & Control & \\
\hline & M2-M0 & & & M6-Mo & & & M12-M0 & & \\
\hline $\begin{array}{l}\text { QOL patient } \\
\text { by caregiver }\end{array}$ & $\begin{array}{l}0.77[-0.13 \\
\text { to } 1.66]\end{array}$ & $\begin{array}{l}0.09[- \\
0.83 \text { to } \\
1.00]\end{array}$ & $\begin{array}{l}0.68[-0.60 \text { to } \\
1.96] \\
p=0.2970\end{array}$ & $\begin{array}{l}0.15[-0.65 \\
\text { to } 0.94]\end{array}$ & $\begin{array}{l}-0.18 \\
{[-0.98} \\
\text { to } 0.62]\end{array}$ & $\begin{array}{l}0.33[-0.80 \text { to } \\
1.46] \\
p=0.5651\end{array}$ & $\begin{array}{l}-0.78[- \\
1.93 \text { to } 0.38]\end{array}$ & $\begin{array}{l}-0.59 \\
{[-1.78} \\
\text { to } 0.61]\end{array}$ & $\begin{array}{l}-0.19[-1.85 \text { to } \\
1.47] \\
p=0.8198\end{array}$ \\
\hline $\begin{array}{l}\text { QOL patient } \\
\text { by patient }\end{array}$ & $\begin{array}{l}0.72[-0.44 \\
\text { to } 1.88]\end{array}$ & $\begin{array}{l}-0.98 \\
{[-2.19} \\
\text { to } 0.24]\end{array}$ & $\begin{array}{l}1.70[0.01 \text { to } 3.38] \\
\boldsymbol{p}=\mathbf{0 . 0 4 8 3}\end{array}$ & $\begin{array}{l}0.96[0 \\
1.91]\end{array}$ & $\begin{array}{l}-0.78 \\
{[-1.80} \\
\text { to } 0.23]\end{array}$ & $\begin{array}{l}1.74[0.34 \text { to } 3.15] \\
\boldsymbol{p}=\mathbf{0 . 0 1 5 4}\end{array}$ & $\begin{array}{l}1.32[0.07 \text { to } \\
2.56]\end{array}$ & $\begin{array}{l}-0.50 \\
{[-1.89} \\
\text { to } 0.89]\end{array}$ & $\begin{array}{l}1.82[-0.06 \text { to } \\
3.69] \\
p=0.0575\end{array}$ \\
\hline PI f*g & $\begin{array}{l}-2.52[- \\
6.32 \text { to } 1.29]\end{array}$ & $\begin{array}{l}0.26[- \\
3.51 \text { to } \\
4.03]\end{array}$ & $\begin{array}{l}-2.77[-8.13 \text { to } \\
2.59] \\
p=0.3090\end{array}$ & $\begin{array}{l}-2.64[- \\
5.90 \text { to } 0.61]\end{array}$ & $\begin{array}{l}1.09[- \\
2.18 \text { to } \\
4.35]\end{array}$ & $\begin{array}{l}-3.73[-8.34 \text { to } \\
0.89] \\
p=0.1126\end{array}$ & $\begin{array}{l}-2.83[- \\
6.89 \text { to } 1.23]\end{array}$ & $\begin{array}{l}2.33[- \\
1.94 \text { to } \\
6.60]\end{array}$ & $\begin{array}{l}-5.16[-11.05 \text { to } \\
0.73] \\
p=0.0859\end{array}$ \\
\hline $\mathrm{DL}$ & $\begin{array}{l}-0.09[- \\
0.20 \text { to } 0.02]\end{array}$ & $\begin{array}{l}-0.15 \\
{[-0.25} \\
\text { to }- \\
0.04]\end{array}$ & $\begin{array}{l}0.06[-0.09 \text { to } \\
0.21] \\
p=0.4519\end{array}$ & $\begin{array}{l}-0.23[- \\
0.32 \text { to }- \\
0.13]\end{array}$ & $\begin{array}{l}-0.24 \\
{[-0.33} \\
\text { to }- \\
0.15]\end{array}$ & $\begin{array}{l}0.02[-0.12 \text { to } \\
0.15] \\
p=0.8150\end{array}$ & $\begin{array}{l}-0.43[- \\
0.59 \text { to }- \\
0.27]\end{array}$ & $\begin{array}{l}-0.38 \\
{[-0.54} \\
\text { to }- \\
0.23]\end{array}$ & $\begin{array}{l}-0.05[-0.27 \text { to } \\
0.18] \\
p=0.6855\end{array}$ \\
\hline $\begin{array}{l}\text { IADL ( } \geq 4 \text { vs } \\
<4 \\
\text { incapacities) }\end{array}$ & $\begin{array}{l}1.60[1.10 \text { to } \\
2.31]\end{array}$ & $\begin{array}{l}1.46 \\
{[1.04 \text { to }} \\
2.06]\end{array}$ & to 1.72$]$ & .34 to & $\begin{array}{l}3.14 \\
{[1.13 \text { to }} \\
8.69]\end{array}$ & o 5.11$]$ & & $\begin{array}{l}9.83 \\
{[1.28 \text { to }} \\
75.52]\end{array}$ & $\begin{array}{l}1.68[0.11 \text { to } \\
26.12] \\
p=0.7096\end{array}$ \\
\hline AMSE & $\begin{array}{l}-0.38[- \\
0.95 \text { to } 0.19]\end{array}$ & $\begin{array}{l}-0.76 \\
{[-1.32} \\
\text { to }- \\
0.19]\end{array}$ & $\begin{array}{l}0.38[-0.43 \text { to } \\
1.18] \\
p=0.3560\end{array}$ & $\begin{array}{l}-1.08[- \\
1.63 \text { to }- \\
0.52]\end{array}$ & $\begin{array}{l}-1.07 \\
{[-1.61} \\
\text { to }- \\
0.53]\end{array}$ & $\begin{array}{l}-0.01[-0.79 \text { to } \\
0.77] \\
p=0.9825\end{array}$ & $\begin{array}{l}-2.12[- \\
2.92 \text { to }- \\
1.32]\end{array}$ & $\begin{array}{l}-1.53 \\
{[-2.31} \\
\text { to }- \\
0.76]\end{array}$ & $\begin{array}{l}-0.59[-1.71 \text { to } \\
0.53] \\
p=0.3007\end{array}$ \\
\hline $\begin{array}{l}\text { One leg } \\
\text { balance } \\
\text { (abnormal vs } \\
\text { normal) }\end{array}$ & $\begin{array}{l}1.00[0.87 \text { to } \\
1.14]\end{array}$ & $\begin{array}{l}1.08 \\
{[0.93 \text { to }} \\
1.25]\end{array}$ & $\begin{array}{l}6 \text { to } 1.13] \\
82\end{array}$ & $\begin{array}{l}0.99[0.65 \text { to } \\
1.50]\end{array}$ & $\begin{array}{l}1.26 \\
{[0.82 \text { to }} \\
1.94]\end{array}$ & $\begin{array}{l}3 \text { to } 1.43] \\
82\end{array}$ & $\begin{array}{l}0.98[0.42 \text { to } \\
2.25]\end{array}$ & $\begin{array}{l}1.58 \\
{[0.67 \text { to }} \\
3.76]\end{array}$ & $\begin{array}{l}0.62[0.19 \text { to } 2.04] \\
p=0.4282\end{array}$ \\
\hline Zarit & $\begin{array}{l}-2.38[- \\
4.50 \text { to }- \\
0.25]\end{array}$ & $\begin{array}{l}-0.14 \\
{[-2.24} \\
\text { to } 1.96]\end{array}$ & $\begin{array}{l}-2.24[-5.23 \text { to } \\
0.75] \\
p=0.1411\end{array}$ & $\begin{array}{l}-1.19[- \\
3.18 \text { to } 0.80]\end{array}$ & $\begin{array}{l}0.35[- \\
1.62 \text { to } \\
2.31]\end{array}$ & $\begin{array}{l}-1.54[-4.33 \text { to } \\
1.26] \\
p=0.2786\end{array}$ & $\begin{array}{l}0.58[-2.30 \\
\text { to } 3.46]\end{array}$ & $\begin{array}{l}1.07[- \\
1.79 \text { to } \\
3.92]\end{array}$ & $\begin{array}{l}-0.49[-4.54 \text { to } \\
3.57] \\
p=0.8128\end{array}$ \\
\hline $\begin{array}{l}\text { Mini-GDS ( } \geq \\
1 \text { vs }=0)\end{array}$ & $\begin{array}{l}1.02[0.86 \text { to } \\
1.22]\end{array}$ & $\begin{array}{l}0.89 \\
{[0.74 \text { to }} \\
1.06]\end{array}$ & $\begin{array}{l}1.15[0.89 \text { to } 1.48] \\
p=0.2759\end{array}$ & $\begin{array}{l}1.06[0.63 \text { to } \\
1.80]\end{array}$ & $\begin{array}{l}0.70 \\
{[0.41 \text { to }} \\
1.19]\end{array}$ & $\begin{array}{l}1.52[0.71 \text { to } 3.22] \\
p=0.2759\end{array}$ & $\begin{array}{l}1.13[0.39 \text { to } \\
3.26]\end{array}$ & $\begin{array}{l}0.49 \\
{[0.17 \text { to }} \\
1.43]\end{array}$ & $\begin{array}{l}2.30[0.51 \text { to } \\
10.39] \\
p=0.2759\end{array}$ \\
\hline NHP & $\begin{array}{l}-0.59[- \\
14.48 \text { to } \\
13.30]\end{array}$ & $\begin{array}{l}-6.38 \\
{[-20.02} \\
\text { to } 7.27]\end{array}$ & $\begin{array}{l}5.79[-13.68 \text { to } \\
25.26] \\
p=0.5582\end{array}$ & $\begin{array}{l}2.57[-11.03 \\
\text { to } 16.17]\end{array}$ & $\begin{array}{l}1.95[- \\
11.34 \text { to } \\
15.23]\end{array}$ & $\begin{array}{l}0.62[-18.39 \text { to } \\
19.64] \\
p=0.9484\end{array}$ & $\begin{array}{l}7.31[-12.99 \\
\text { to } 27.60]\end{array}$ & $\begin{array}{l}14.43[- \\
5.37 \text { to } \\
34.23]\end{array}$ & $\begin{array}{l}-7.12[-35.48 \text { to } \\
21.23] \\
p=0.6199\end{array}$ \\
\hline
\end{tabular}

${ }^{a}$ Mean $(95 \% \mathrm{Cl})$ : estimated with the mean values at baseline

bodds ratio $(95 \% \mathrm{Cl})$

$[50,58,59]$. However, the internal validity of the QOL-AD scale from mild to moderately severe stages of the disease has been formally validated, allowed us to pursue this goal [35].

Regarding the intervention itself, although we created an educational intervention close to the effective multicomponent strategies in terms of content and duration, and assessed it a qualitative manner (fulfilment of educational objectives on visual analogue scale), the dyadic perspective remained challenging. Indeed we tried to help each dyad reach their own individual goals through the acquirement by the caregiver of knowledge and skills. At the end of the programme, caregivers were asked to rate their goals as "achieved" or "to reach" and to estimate skills as "acquired" or "to strengthen" on visual analogue scales. If the educational intervention can reinforce the carer in his or her role and bring about behavioural changes, then measuring any change in the way he or she provides care and, consequently, the potential impact on patient health is complex.

Moreover, the intervention needs to be standardized but also sufficiently tailored to dyadic issues. During the 


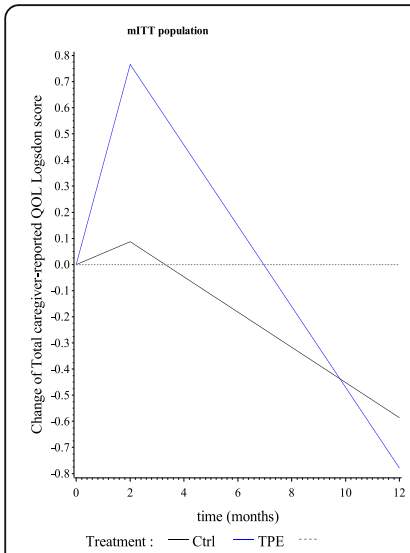

A: Patient's QOL caregiver reported

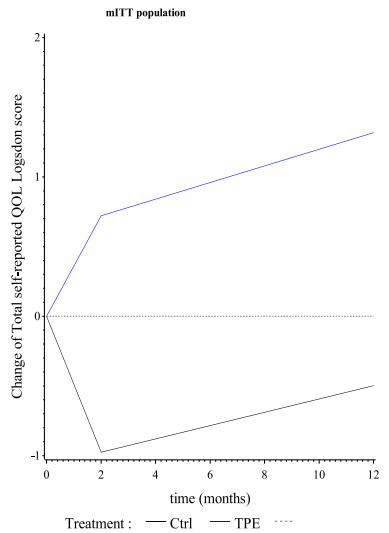

A': Patient's QOL self-reported

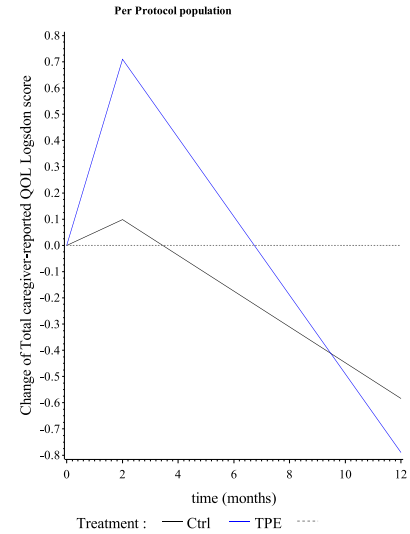

B: Patient's QOL caregiver reported

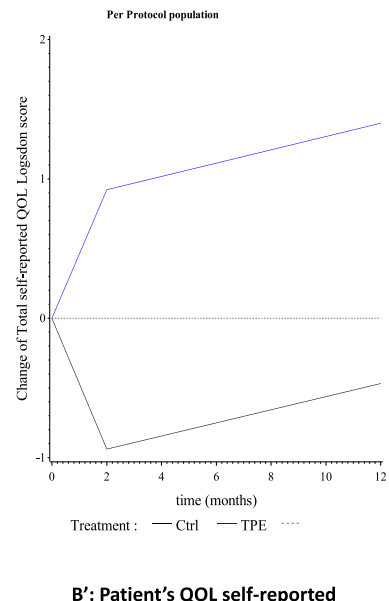

B': Patient's QOL self-reported

Fig. 3 Change from baseline over time in patients' $Q O L$ caregiver-reported and self-reported by patients in the $m / T T(n=155)\left(\mathbf{A}, \mathbf{A}^{\prime}\right)$ and PP $(n=$ 148) (B, B') populations

Table 3 Effect of the THERAD intervention vs control on patient's QOL caregiver-reported and self-reported: sensitivity analysis in per protocol population and/or with another mixed linear model method

\begin{tabular}{|c|c|c|c|c|c|c|c|c|c|}
\hline \multirow{3}{*}{ Outcome } & \multicolumn{2}{|c|}{$\begin{array}{l}\text { Estimated change } \\
\text { from baseline } \\
\text { mean }^{\mathrm{a}}(95 \% \mathrm{Cl})\end{array}$} & \multirow{2}{*}{$\begin{array}{l}\text { Estimated } \\
\text { differences in } \\
\text { change from } \\
\text { baseline } \\
\text { mean }(95 \% \mathrm{Cl}) \\
p \text { value } \\
\text { Intervention vs } \\
\text { Control }\end{array}$} & \multicolumn{2}{|c|}{$\begin{array}{l}\text { Estimated change } \\
\text { from baseline } \\
\text { mean }^{a}(95 \% C \mathrm{C})\end{array}$} & \multirow{3}{*}{$\begin{array}{l}\text { Estimated } \\
\text { differences in } \\
\text { change from } \\
\text { baseline } \\
\text { mean }(95 \% \mathrm{CI}) \\
p \text { value } \\
\text { Intervention vs } \\
\text { Control }\end{array}$} & \multicolumn{2}{|c|}{$\begin{array}{l}\text { Estimated change } \\
\text { from baseline } \\
\text { mean }^{\mathrm{a}}(95 \% \mathrm{Cl})\end{array}$} & \multirow{2}{*}{$\begin{array}{l}\text { Estimated } \\
\text { differences in } \\
\text { change from } \\
\text { baseline } \\
\text { mean }(95 \% \mathrm{Cl}) \\
p \text { value } \\
\text { Intervention vs } \\
\text { Control }\end{array}$} \\
\hline & Intervention & Control & & Intervention & Control & & Intervention & Control & \\
\hline & \multicolumn{2}{|l|}{$\mathrm{M} 2-\mathrm{MO}$} & & \multicolumn{2}{|l|}{ M6-Mo } & & \multicolumn{2}{|l|}{ M12-M0 } & \\
\hline \multicolumn{10}{|c|}{ Per-protocol population model 1} \\
\hline $\begin{array}{l}\text { QOL patient by } \\
\text { caregiver (int: } 1 \\
=72, \text { ctrl: } n=76 \text { ) }\end{array}$ & $\begin{array}{l}0.71[-0.23 \\
\text { to } 1.65]\end{array}$ & $\begin{array}{l}0.10[- \\
0.82 \text { to } \\
1.01]\end{array}$ & $\begin{array}{l}0.61[-0.70 \text { to } \\
1.93] \\
p=0.3595\end{array}$ & $\begin{array}{l}0.11[-0.72 \\
\text { to } 0.94]\end{array}$ & $\begin{array}{l}-0.17 \\
{[-0.98} \\
\text { to } 0.63]\end{array}$ & $\begin{array}{l}0.28[-0.88 \text { to } \\
1.45] \\
p=0.6287\end{array}$ & $\begin{array}{l}-0.79[- \\
1.96 \text { to } 0.38]\end{array}$ & $\begin{array}{l}-0.58 \\
{[-1.78} \\
\text { to } 0.61]\end{array}$ & $\begin{array}{l}-0.21[-1.88 \text { to } \\
1.47] \\
p=0.8086\end{array}$ \\
\hline $\begin{array}{l}\text { QOL patient by } \\
\text { patient (int: } n= \\
56, \text { ctrl: } n=53 \text { ) }\end{array}$ & $\begin{array}{l}0.92[-0.30 \\
\text { to } 2.14]\end{array}$ & $\begin{array}{l}-0.94 \\
{[-2.17} \\
\text { to } 0.29]\end{array}$ & $\begin{array}{l}1.86[0.13 \text { to } \\
3.60] \\
\boldsymbol{p}=\mathbf{0 . 0 3 5 6}\end{array}$ & $\begin{array}{l}1.11[0.11 \text { to } \\
2.11]\end{array}$ & $\begin{array}{l}-0.75 \\
{[-1.78} \\
\text { to } 0.28]\end{array}$ & $\begin{array}{l}1.86[0.42 \text { to } \\
3.31] \\
\boldsymbol{p}=\mathbf{0 . 0 1 1 8}\end{array}$ & $\begin{array}{l}1.40[0.14 \text { to } \\
2.66]\end{array}$ & $\begin{array}{l}-0.47 \\
{[-1.87} \\
\text { to } 0.93]\end{array}$ & $\begin{array}{l}1.87[-0.02 \text { to } \\
3.76] \\
p=0.0529\end{array}$ \\
\hline \multicolumn{10}{|c|}{ Per-protocol population model 2} \\
\hline $\begin{array}{l}\text { QOL patient by } \\
\text { caregiver (int: } n \\
=73, \text { ctrl: } n=90 \text { ) }\end{array}$ & $\begin{array}{l}-0.18[- \\
0.38 \text { to } 0.02]\end{array}$ & $\begin{array}{l}-0.06 \\
{[-0.26} \\
\text { to } 0.15]\end{array}$ & $\begin{array}{l}-0.12[-0.41 \text { to } \\
0.16] \\
p=0.4023\end{array}$ & $\begin{array}{l}-0.53[- \\
1.14 \text { to } 0.07]\end{array}$ & $\begin{array}{l}-0.17 \\
{[-0.78} \\
\text { to } 0.44]\end{array}$ & $\begin{array}{l}-0.36[-1.22 \text { to } \\
0.49] \\
p=0.4023\end{array}$ & $\begin{array}{l}-1.07[- \\
2.27 \text { to } 0.14]\end{array}$ & $\begin{array}{l}-0.34 \\
{[-1.56} \\
\text { to } 0.88]\end{array}$ & $\begin{array}{l}-0.73[-2.45 \text { to } \\
0.99] \\
p=0.4023\end{array}$ \\
\hline $\begin{array}{l}\text { QOL patient by } \\
\text { patient (int: } n= \\
61, \text { ctrl: } n=67 \text { ) }\end{array}$ & $\begin{array}{l}0.21[-0.01 \\
\text { to } 0.43]\end{array}$ & $\begin{array}{l}-0.06 \\
{[-0.31} \\
\text { to } 0.18]\end{array}$ & $\begin{array}{l}0.28[-0.05 \text { to } \\
0.60] \\
p=0.0953\end{array}$ & $\begin{array}{l}0.64[-0.02 \\
\text { to } 1.29]\end{array}$ & $\begin{array}{l}-0.19 \\
{[-0.92} \\
\text { to } 0.53]\end{array}$ & $\begin{array}{l}0.83[-0.15 \text { to } \\
1.81] \\
p=0.0953\end{array}$ & $\begin{array}{l}1.27[-0.04 \\
\text { to } 2.59]\end{array}$ & $\begin{array}{l}-0.39 \\
{[-1.84} \\
\text { to } 1.06]\end{array}$ & $\begin{array}{l}1.66[-0.29 \text { to } \\
3.62] \\
p=0.0953\end{array}$ \\
\hline \multicolumn{10}{|c|}{ ITT population model 2} \\
\hline $\begin{array}{l}\text { QOL patient by } \\
\text { caregiver (int: } n \\
=95, \text { ctrl: } n=90 \text { ) }\end{array}$ & $\begin{array}{l}-0.18[- \\
0.37 \text { to } 0.02]\end{array}$ & $\begin{array}{l}-0.06 \\
{[-0.26} \\
\text { to } 0.15]\end{array}$ & $\begin{array}{l}-0.12[-0.40 \text { to } \\
0.16] \\
p=0.4005\end{array}$ & $\begin{array}{l}-0.53[- \\
1.12 \text { to } 0.06]\end{array}$ & $\begin{array}{l}-0.17 \\
{[-0.78} \\
\text { to } 0.44]\end{array}$ & $\begin{array}{l}-0.36[-1.21 \text { to } \\
0.49] \\
p=0.4005\end{array}$ & $\begin{array}{l}-1.06[- \\
2.24 \text { to } 0.12]\end{array}$ & $\begin{array}{l}-0.33 \\
{[-1.55} \\
\text { to } 0.88]\end{array}$ & $\begin{array}{l}-0.72[-2.42 \text { to } \\
0.97] p=0.4005\end{array}$ \\
\hline $\begin{array}{l}\text { QOL patient by } \\
\text { patient (int: } n= \\
78, \text { ctrl: } n=67 \text { ) }\end{array}$ & $\begin{array}{l}0.22[0.00 \text { to } \\
0.43]\end{array}$ & $\begin{array}{l}-0.06 \\
{[-0.31} \\
\text { to } 0.18]\end{array}$ & $\begin{array}{l}0.28[-0.04 \text { to } \\
0.61] \\
p=0.0858\end{array}$ & $\begin{array}{l}0.65[0.01 \text { to } \\
1.30]\end{array}$ & $\begin{array}{l}-0.19 \\
{[-0.92} \\
\text { to } 0.53]\end{array}$ & $\begin{array}{l}0.85[-0.12 \text { to } \\
1.82] \\
p=0.0858\end{array}$ & $\begin{array}{l}1.31[0.02 \text { to } \\
2.60]\end{array}$ & $\begin{array}{l}-0.39 \\
{[-1.84} \\
\text { to } 1.06]\end{array}$ & $\begin{array}{l}1.70[-0.24 \text { to } \\
3.63] \\
p=0.0858\end{array}$ \\
\hline
\end{tabular}

Model 1: linear mixed model adjusted on baseline data

Model 2: linear mixed model with baseline value included in the dependent variable

${ }^{a}$ Mean $(95 \% \mathrm{Cl})$ : estimated with the mean values at baseline for model 1 
Table 4 Patient's QOL caregiver-reported and self-reported in subgroup analysis of the THERAD study (results from linear mixed models in modified intention-to-treat population). 'Mean (95\%Cl): estimated with the mean values at baseline

\begin{tabular}{|c|c|c|c|c|c|c|c|c|c|c|}
\hline \multirow[b]{3}{*}{ Outcome } & \multirow[b]{3}{*}{ Subgroup } & \multicolumn{2}{|c|}{$\begin{array}{l}\text { Estimated change } \\
\text { from baseline } \\
\text { mean }^{1}(95 \% \mathrm{Cl})\end{array}$} & \multirow{2}{*}{$\begin{array}{l}\text { Estimated } \\
\text { differences in } \\
\text { change from } \\
\text { baseline } \\
\text { mean }(95 \% \mathrm{Cl}) \\
p \text { value } \\
\text { Intervention } \\
\text { vs Control }\end{array}$} & \multicolumn{2}{|c|}{$\begin{array}{l}\text { Estimated change } \\
\text { from baseline } \\
\text { mean }^{1}(95 \% \mathrm{Cl})\end{array}$} & \multirow{3}{*}{$\begin{array}{l}\text { Estimated } \\
\text { differences in } \\
\text { change from } \\
\text { baseline } \\
\text { mean }(95 \% \mathrm{Cl}) \\
p \text { value } \\
\text { Intervention } \\
\text { vs Control }\end{array}$} & \multicolumn{2}{|c|}{$\begin{array}{l}\text { Estimated change } \\
\text { from baseline } \\
\text { mean }^{1}(95 \% \mathrm{Cl})\end{array}$} & \multirow{2}{*}{$\begin{array}{l}\text { Estimated } \\
\text { differences in } \\
\text { change from } \\
\text { baseline } \\
\text { mean }(95 \% \mathrm{Cl}) \\
p \text { value } \\
\text { Intervention } \\
\text { vs Control }\end{array}$} \\
\hline & & Intervention & Control & & Intervention & Control & & Intervention & Control & \\
\hline & & M2-M0 & & & M6-Mo & & & M12-M0 & & \\
\hline $\begin{array}{l}\text { Patient's } \\
\text { QOL } \\
\text { caregiver- } \\
\text { reported }\end{array}$ & $\begin{array}{l}\text { 1. tot } \\
\text { Zarit: }>40\end{array}$ & $\begin{array}{l}0.33[-1.48 \\
\text { to } 2.14]\end{array}$ & $\begin{array}{l}-0.74 \\
{[-2.40} \\
\text { to } 0.92]\end{array}$ & $\begin{array}{l}1.07[-1.32 \text { to } \\
3.45] \\
p=0.3783\end{array}$ & $\begin{array}{l}0.92[-0.71 \\
\text { to } 2.56]\end{array}$ & $\begin{array}{l}-1.02 \\
{[-2.48} \\
\text { to } 0.44]\end{array}$ & $\begin{array}{l}1.95[-0.17 \text { to } \\
4.06] p= \\
0.0709\end{array}$ & $\begin{array}{l}1.82[-0.70 \\
\text { to } 4.34]\end{array}$ & $\begin{array}{l}-1.45 \\
{[-3.66} \\
\text { to } 0.76]\end{array}$ & $\begin{array}{l}3.27[-0.03 \text { to } \\
6.57] \\
p=0.0523\end{array}$ \\
\hline $\begin{array}{l}\text { Patient's } \\
\text { QOL } \\
\text { caregiver- } \\
\text { reported }\end{array}$ & $\begin{array}{l}\text { 2. tot } \\
\text { Zarit: [20; } \\
40]\end{array}$ & $\begin{array}{l}1.31[-0.09 \\
\text { to } 2.70]\end{array}$ & $\begin{array}{l}-0.34 \\
{[-1.72} \\
\text { to } 1.03]\end{array}$ & $\begin{array}{l}1.65[-0.31 \text { to } \\
3.61] \\
p=0.0985\end{array}$ & $\begin{array}{l}0.21[-0.99 \\
\text { to } 1.41]\end{array}$ & $\begin{array}{l}-0.61 \\
{[-1.81} \\
\text { to } 0.60]\end{array}$ & $\begin{array}{l}0.82[-0.88 \text { to } \\
2.51] \\
p=0.3438\end{array}$ & $\begin{array}{l}-1.44[- \\
3.06 \text { to } 0.19]\end{array}$ & $\begin{array}{l}-1.00 \\
{[-2.75} \\
\text { to } 0.75]\end{array}$ & $\begin{array}{l}-0.44[-2.83 \\
\text { to } 1.95] \\
p=0.7185\end{array}$ \\
\hline $\begin{array}{l}\text { Patient's } \\
\text { QOL } \\
\text { caregiver- } \\
\text { reported }\end{array}$ & $\begin{array}{l}\text { 3. tot } \\
\text { Zarit: } \leq 20\end{array}$ & $\begin{array}{l}0.45[-1.10 \\
\text { to } 2.00]\end{array}$ & $\begin{array}{l}2.00 \\
{[0.17 \text { to }} \\
3.84]\end{array}$ & $\begin{array}{l}-1.55[-3.88 \\
\text { to } 0.78] \\
p=0.1898\end{array}$ & $\begin{array}{l}-0.20[- \\
1.61 \text { to } 1.20]\end{array}$ & $\begin{array}{l}1.58 \\
{[0.00 \text { to }} \\
3.16]\end{array}$ & $\begin{array}{l}-1.78[-3.81 \\
\text { to } 0.24] \\
p=0.0835\end{array}$ & $\begin{array}{l}-1.18[- \\
3.17 \text { to } 0.80]\end{array}$ & $\begin{array}{l}0.94[- \\
1.24 \text { to } \\
3.13]\end{array}$ & $\begin{array}{l}-2.13[-5.01 \\
\text { to } 0.76] \\
p=0.1468\end{array}$ \\
\hline $\begin{array}{l}\text { Patient's } \\
\text { QOL } \\
\text { caregiver- } \\
\text { reported }\end{array}$ & $\begin{array}{l}\text { 4. tot } \\
\text { Zarit: }>40 \\
\text { vs } \leq 20\end{array}$ & & & $\begin{array}{l}2.62[-0.71 \text { to } \\
5.95] \\
p=0.1226\end{array}$ & & & $\begin{array}{l}3.73[0.80 \text { to } \\
6.66] \\
\boldsymbol{p}=\mathbf{0 . 0 1 2 9 *}\end{array}$ & & & $\begin{array}{l}5.40[1.01 \text { to } \\
9.79] \\
\boldsymbol{p}=\mathbf{0 . 0 1 6 3 *}\end{array}$ \\
\hline $\begin{array}{l}\text { Patient's } \\
\text { QOL } \\
\text { caregiver- } \\
\text { reported }\end{array}$ & $\begin{array}{l}\text { 5. tot } \\
\text { Zarit: [20; } \\
40] \text { vs } \leq \\
20\end{array}$ & & & $\begin{array}{l}3.20[0.16 \text { to } \\
6.24] \\
\boldsymbol{p}=\mathbf{0 . 0 3 9 0 *}\end{array}$ & & & $\begin{array}{l}2.60[-0.03 \text { to } \\
5.23] \\
p=0.0530\end{array}$ & & & $\begin{array}{l}1.69[-2.05 \text { to } \\
5.43] \\
p=0.3719\end{array}$ \\
\hline $\begin{array}{l}\text { Patient's } \\
\text { QOL } \\
\text { caregiver- } \\
\text { reported }\end{array}$ & $\begin{array}{l}\text { 1. tot } \\
\text { MMS: } \leq \\
15\end{array}$ & $\begin{array}{l}1.33[-0.36 \\
\text { to } 3.02]\end{array}$ & $\begin{array}{l}0.14[- \\
1.44 \text { to } \\
1.72]\end{array}$ & $\begin{array}{l}1.19[-1.12 \text { to } \\
3.50] \\
p=0.3112\end{array}$ & $\begin{array}{l}0.60[-0.94 \\
\text { to } 2.14]\end{array}$ & $\begin{array}{l}-0.25 \\
{[-1.60} \\
\text { to } 1.11]\end{array}$ & $\begin{array}{l}0.85[-1.21 \text { to } \\
2.90] \\
p=0.4158\end{array}$ & $\begin{array}{l}-0.49[- \\
2.78 \text { to } 1.80]\end{array}$ & $\begin{array}{l}-0.83 \\
{[-2.86} \\
\text { to } 1.21]\end{array}$ & $\begin{array}{l}0.33[-2.73 \text { to } \\
3.40] \\
p=0.8296\end{array}$ \\
\hline $\begin{array}{l}\text { Patient's } \\
\text { QOL } \\
\text { caregiver- } \\
\text { reported }\end{array}$ & $\begin{array}{l}\text { 2. tot } \\
\text { MMS: [15; } \\
\text { 20] }\end{array}$ & $\begin{array}{l}1.08[-0.34 \\
\text { to } 2.51]\end{array}$ & $\begin{array}{l}0.36[- \\
1.04 \text { to } \\
1.75]\end{array}$ & $\begin{array}{l}0.73[-1.26 \text { to } \\
2.72] \\
p=0.4713\end{array}$ & $\begin{array}{l}0.59[-0.67 \\
\text { to } 1.84]\end{array}$ & $\begin{array}{l}-0.05 \\
{[-1.28} \\
\text { to } 1.18]\end{array}$ & $\begin{array}{l}0.63[-1.12 \text { to } \\
2.39] \\
p=0.4781\end{array}$ & $\begin{array}{l}-0.16[- \\
1.96 \text { to } 1.64]\end{array}$ & $\begin{array}{l}-0.65 \\
{[-2.44} \\
\text { to } 1.14]\end{array}$ & $\begin{array}{l}0.49[-2.05 \text { to } \\
3.02] \\
p=0.7059\end{array}$ \\
\hline $\begin{array}{l}\text { Patient's } \\
\text { QOL } \\
\text { caregiver- } \\
\text { reported }\end{array}$ & $\begin{array}{l}\text { 3. tot } \\
\text { MMS: }>20\end{array}$ & $\begin{array}{l}-0.08[- \\
1.62 \text { to } 1.47]\end{array}$ & $\begin{array}{l}-0.49 \\
{[-2.39} \\
\text { to } 1.41]\end{array}$ & $\begin{array}{l}0.41[-2.04 \text { to } \\
2.86] \\
p=0.7413\end{array}$ & $\begin{array}{l}-0.72[- \\
2.07 \text { to } 0.63]\end{array}$ & $\begin{array}{l}-0.33 \\
{[-2.02} \\
\text { to } 1.37]\end{array}$ & $\begin{array}{l}-0.39[-2.56 \\
\text { to } 1.77] \\
p=0.7198\end{array}$ & $\begin{array}{l}-1.68[- \\
3.62 \text { to } 0.25]\end{array}$ & $\begin{array}{l}-0.08 \\
{[-2.59} \\
\text { to } 2.43]\end{array}$ & $\begin{array}{l}-1.60[-4.77 \\
\text { to } 1.57] \\
p=0.3190\end{array}$ \\
\hline $\begin{array}{l}\text { Patient's } \\
\mathrm{QOL} \\
\text { caregiver- } \\
\text { reported }\end{array}$ & $\begin{array}{l}\text { 4. tot } \\
\text { MMS: } \leq \\
15 \text { vs }>20\end{array}$ & & & $\begin{array}{l}0.78[-2.62 \text { to } \\
4.18] \\
p=0.6518\end{array}$ & & & $\begin{array}{l}1.24[-1.77 \text { to } \\
4.26] \\
p=0.4167\end{array}$ & & & $\begin{array}{l}1.94[-2.49 \text { to } \\
6.36] \\
p=0.3882\end{array}$ \\
\hline $\begin{array}{l}\text { Patient's } \\
\text { QOL } \\
\text { caregiver- } \\
\text { reported }\end{array}$ & $\begin{array}{l}\text { 5. tot } \\
\text { MMS: [15; } \\
\text { 20] vs > } \\
20\end{array}$ & & & $\begin{array}{l}0.32[-2.86 \text { to } \\
3.49] \\
p=0.8435\end{array}$ & & & $\begin{array}{l}1.03[-1.78 \text { to } \\
3.83] \\
p=0.4710\end{array}$ & & & $\begin{array}{l}2.09[-1.98 \text { to } \\
6.16] \\
p=0.3123\end{array}$ \\
\hline $\begin{array}{l}\text { Self- } \\
\text { reported } \\
\text { QOL }\end{array}$ & $\begin{array}{l}\text { 1. tot } \\
\text { Zarit: }>40\end{array}$ & $\begin{array}{l}0.56[-1.76 \\
\text { to } 2.88]\end{array}$ & $\begin{array}{l}-4.13 \\
{[-6.60} \\
\text { to }- \\
1.66]\end{array}$ & $\begin{array}{l}4.69[1.30 \text { to } \\
8.08] \\
\boldsymbol{p}=\mathbf{0 . 0 0 6 9 *}\end{array}$ & $\begin{array}{l}1.33[-0.62 \\
\text { to } 3.27]\end{array}$ & $\begin{array}{l}-2.99 \\
{[-4.94} \\
\text { to }- \\
1.03]\end{array}$ & $\begin{array}{l}4.31[1.54 \text { to } \\
7.08] \\
\boldsymbol{p}=\mathbf{0 . 0 0 2 6 *}^{*}\end{array}$ & $\begin{array}{l}2.47[-0.17 \\
\text { to } 5.11]\end{array}$ & $\begin{array}{l}-1.27 \\
{[-4.56} \\
\text { to } 2.02]\end{array}$ & $\begin{array}{l}3.74[-0.49 \text { to } \\
7.98] \\
p=0.0830\end{array}$ \\
\hline $\begin{array}{l}\text { Self- } \\
\text { reported } \\
\text { QOL }\end{array}$ & $\begin{array}{l}\text { 2. tot } \\
\text { Zarit: [20; } \\
40]\end{array}$ & $\begin{array}{l}1.13[-0.69 \\
\text { to } 2.94]\end{array}$ & $\begin{array}{l}0.52[- \\
1.28 \text { to } \\
2.31]\end{array}$ & $\begin{array}{l}0.61[-1.94 \text { to } \\
3.15] \\
p=0.6383\end{array}$ & $\begin{array}{l}1.22[-0.24 \\
\text { to } 2.68]\end{array}$ & $\begin{array}{l}0.40[- \\
1.10 \text { to } \\
1.90]\end{array}$ & $\begin{array}{l}0.82[-1.26 \text { to } \\
2.90] \\
p=0.4368\end{array}$ & $\begin{array}{l}1.37[-0.52 \\
\text { to } 3.26]\end{array}$ & $\begin{array}{l}0.23[- \\
1.74 \text { to } \\
2.20]\end{array}$ & $\begin{array}{l}1.14[-1.58 \text { to } \\
3.86] \\
p=0.4102\end{array}$ \\
\hline $\begin{array}{l}\text { Self- } \\
\text { reported } \\
\text { QOL }\end{array}$ & $\begin{array}{l}\text { 3. tot } \\
\text { Zarit: } \leq 20\end{array}$ & $\begin{array}{l}0.42[-1.43 \\
\text { to } 2.28]\end{array}$ & $\begin{array}{l}-0.66 \\
{[-2.76} \\
\text { to } 1.44]\end{array}$ & $\begin{array}{l}1.08[-1.70 \text { to } \\
3.86] \\
p=0.4428\end{array}$ & $\begin{array}{l}0.51[-1.02 \\
\text { to } 2.05]\end{array}$ & $\begin{array}{l}-0.56 \\
{[-2.32} \\
\text { to } 1.20]\end{array}$ & $\begin{array}{l}1.08[-1.24 \text { to } \\
3.39] \\
p=0.3580\end{array}$ & $\begin{array}{l}0.64[-1.35 \\
\text { to } 2.64]\end{array}$ & $\begin{array}{l}-0.42 \\
{[-2.73} \\
\text { to } 1.89]\end{array}$ & $\begin{array}{l}1.06[-1.97 \text { to } \\
4.10] \\
p=0.4908\end{array}$ \\
\hline Self- & 4. tot & & & $3.61[-0.77$ to & & & $3.24[-0.36$ to & & & $2.68[-2.51$ to \\
\hline
\end{tabular}


Table 4 Patient's QOL caregiver-reported and self-reported in subgroup analysis of the THERAD study (results from linear mixed models in modified intention-to-treat population). ${ }^{1}$ Mean (95\%Cl): estimated with the mean values at baseline (Continued)

\begin{tabular}{|c|c|c|c|c|c|c|c|c|c|c|}
\hline \multirow[b]{3}{*}{ Outcome } & \multirow[b]{3}{*}{ Subgroup } & \multicolumn{2}{|c|}{$\begin{array}{l}\text { Estimated change } \\
\text { from baseline } \\
\text { mean }^{1}(95 \% \mathrm{Cl})\end{array}$} & \multirow{2}{*}{$\begin{array}{l}\text { Estimated } \\
\text { differences in } \\
\text { change from } \\
\text { baseline } \\
\text { mean }(95 \% \mathrm{Cl}) \\
p \text { value } \\
\text { Intervention } \\
\text { vs Control }\end{array}$} & \multicolumn{2}{|c|}{$\begin{array}{l}\text { Estimated change } \\
\text { from baseline } \\
\text { mean }^{1}(95 \% \mathrm{Cl})\end{array}$} & \multirow{2}{*}{$\begin{array}{l}\text { Estimated } \\
\text { differences in } \\
\text { change from } \\
\text { baseline } \\
\text { mean }(95 \% \mathrm{Cl}) \\
p \text { value } \\
\text { Intervention } \\
\text { vs Control }\end{array}$} & \multicolumn{2}{|c|}{$\begin{array}{l}\text { Estimated change } \\
\text { from baseline } \\
\text { mean }^{1}(95 \% \mathrm{Cl})\end{array}$} & \multirow{2}{*}{$\begin{array}{l}\text { Estimated } \\
\text { differences in } \\
\text { change from } \\
\text { baseline } \\
\text { mean }(95 \% \mathrm{Cl}) \\
p \text { value } \\
\text { Intervention } \\
\text { vs Control }\end{array}$} \\
\hline & & Intervention & Control & & Intervention & Control & & Intervention & Control & \\
\hline & & M2-MO & & & M6-Mo & & & M12-M0 & & \\
\hline $\begin{array}{l}\text { reported } \\
\text { QOL }\end{array}$ & $\begin{array}{l}\text { Zarit: }>40 \\
\text { vs } \leq 20\end{array}$ & & & $\begin{array}{l}7.99] \\
p=0.1058\end{array}$ & & & $\begin{array}{l}6.83] \\
p=0.0769\end{array}$ & & & $\begin{array}{l}7.86] \\
p=0.3101\end{array}$ \\
\hline $\begin{array}{l}\text { Self- } \\
\text { reported } \\
\text { QOL }\end{array}$ & $\begin{array}{l}\text { 5. tot } \\
\text { Zarit: [20; } \\
40] \text { vs } \leq \\
20\end{array}$ & & & $\begin{array}{l}-0.48[-4.24 \\
\text { to } 3.29] \\
p=0.8030\end{array}$ & & & $\begin{array}{l}-0.26[-3.36 \\
\text { to } 2.85] \\
p=0.8705\end{array}$ & & & $\begin{array}{l}0.08[-3.99 \text { to } \\
4.14] \\
p=0.9706\end{array}$ \\
\hline $\begin{array}{l}\text { Self- } \\
\text { reported } \\
\text { QOL }\end{array}$ & $\begin{array}{l}\text { 1. tot } \\
\text { MMS: } \leq \\
15\end{array}$ & $\begin{array}{l}1.17[-1.33 \\
\text { to } 3.67]\end{array}$ & $\begin{array}{l}-1.30 \\
{[-4.10} \\
\text { to } 1.49]\end{array}$ & $\begin{array}{l}2.47[-1.27 \text { to } \\
6.22] \\
p=0.1947\end{array}$ & $\begin{array}{l}1.52[-0.54 \\
\text { to } 3.58]\end{array}$ & $\begin{array}{l}-0.60 \\
{[-3.05} \\
\text { to } 1.84]\end{array}$ & $\begin{array}{l}2.12[-1.07 \text { to } \\
5.32] \\
p=0.1910\end{array}$ & $\begin{array}{l}2.05[-0.79 \\
\text { to } 4.89]\end{array}$ & $\begin{array}{l}0.44[- \\
3.82 \text { to } \\
4.71]\end{array}$ & $\begin{array}{l}1.61[-3.52 \text { to } \\
6.74] \\
p=0.5381\end{array}$ \\
\hline $\begin{array}{l}\text { Self- } \\
\text { reported } \\
\text { QOL }\end{array}$ & $\begin{array}{l}\text { 2. tot } \\
\text { MMS: [15; } \\
\text { 20] }\end{array}$ & $\begin{array}{l}1.65[-0.19 \\
\text { to } 3.48]\end{array}$ & $\begin{array}{l}-1.06 \\
{[-2.78} \\
\text { to } 0.67]\end{array}$ & $\begin{array}{l}2.70[0.18 \text { to } \\
5.23] \\
\boldsymbol{p}=\mathbf{0 . 0 3 6 0 *}\end{array}$ & $\begin{array}{l}1.63[0.12 \text { to } \\
3.13]\end{array}$ & $\begin{array}{l}-0.92 \\
{[-2.34} \\
\text { to } 0.49]\end{array}$ & $\begin{array}{l}2.55[0.47 \text { to } \\
4.63] \\
\boldsymbol{p}=\mathbf{0 . 0 1 6 6 *}\end{array}$ & $\begin{array}{l}1.60[-0.30 \\
\text { to } 3.49]\end{array}$ & $\begin{array}{l}-0.72 \\
{[-2.57} \\
\text { to } 1.13]\end{array}$ & $\begin{array}{l}2.32[-0.34 \text { to } \\
4.97] \\
p=0.0872\end{array}$ \\
\hline $\begin{array}{l}\text { Self- } \\
\text { reported } \\
\text { QOL }\end{array}$ & $\begin{array}{l}\text { 3. tot } \\
\text { MMS: }>20\end{array}$ & $\begin{array}{l}-0.41[- \\
2.22 \text { to } 1.40]\end{array}$ & $\begin{array}{l}-0.69 \\
{[-2.80} \\
\text { to } 1.42]\end{array}$ & $\begin{array}{l}0.28[-2.50 \text { to } \\
3.06] \\
p=0.8432\end{array}$ & $\begin{array}{l}0.04[-1.45 \\
\text { to } 1.53]\end{array}$ & $\begin{array}{l}-0.55 \\
{[-2.30} \\
\text { to } 1.21]\end{array}$ & $\begin{array}{l}0.59[-1.72 \text { to } \\
2.89] \\
p=0.6140\end{array}$ & $\begin{array}{l}0.73[-1.26 \\
\text { to } 2.72]\end{array}$ & $\begin{array}{l}-0.33 \\
{[-2.69} \\
\text { to } 2.04]\end{array}$ & $\begin{array}{l}1.05[-2.04 \text { to } \\
4.15] \\
p=0.5036\end{array}$ \\
\hline $\begin{array}{l}\text { Self- } \\
\text { reported } \\
\text { QOL }\end{array}$ & $\begin{array}{l}\text { 4. tot } \\
\text { MMS: } \leq \\
15 \text { vs }>20\end{array}$ & & & $\begin{array}{l}2.19[-2.48 \text { to } \\
6.86] \\
p=0.3556\end{array}$ & & & $\begin{array}{l}1.54[-2.42 \text { to } \\
5.49] \\
p=0.4434\end{array}$ & & & $\begin{array}{l}0.55[-5.46 \text { to } \\
6.57] \\
p=0.8563\end{array}$ \\
\hline $\begin{array}{l}\text { Self- } \\
\text { reported } \\
\text { QOL }\end{array}$ & $\begin{array}{l}\text { 5. tot } \\
\text { MMS: [15; } \\
\text { 20] vs > } \\
20\end{array}$ & & & $\begin{array}{l}2.43[-1.32 \text { to } \\
6.17] \\
p=0.2029\end{array}$ & & & $\begin{array}{l}1.96[-1.12 \text { to } \\
5.04] \\
p=0.2097\end{array}$ & & & $\begin{array}{l}1.26[-2.79 \text { to } \\
5.31] \\
p=0.5394\end{array}$ \\
\hline
\end{tabular}

trial, we observed a number of issues between spouse and child caregivers that are known to be different [60]. We noted that an intervention such as ours designed to provide knowledge and skills could be more effective for spouses than child caregivers (the latter may benefit from interventions designed to alleviate the mental load and burden), as suggested in the literature [9]. It should be noted that the programme was of short duration and the intensity was quite low, while TPE is a continuous process that should be continued and adjusted to the disease course and patient lifestyle. However, previous studies assessing interventions of longer durations (312 months) were negative $[18,19]$.

Another interpretation of our contrasting results is possible since self-reported patients' QOL was significantly increased by TPE. Indeed, we cannot exclude that the intervention may have had a positive effect on the patient that was not perceived by his or her caregiver. We hypothesize that the self-rated QOL is closer to reality in the THERAD population. Indeed, the positive significant effect on self-reported QOL at 2 months and 6 months does not remain at 12 months, whereas selfreported QOL is stable in the literature $[50,53]$ in this specific population of patients between mild and moderately severe $A D$, suggesting a possible early and timelimited effect. This stability described in the literature [44] has been attributed to the patient's reduced abilities to estimate any change on a scale $[50,53]$ and, for some authors, to the conceptual nature of certain items (selfesteem) not being understood and being responsible for the missing data beyond the mild stages. Moreover, a decline in the patient's QOL during the mild stages is related to cognitive impairment $[50,58,59]$, as previously note, on which educational interventions have not demonstrated any impact [19]. Last, our subgroup analyses reported an increased intervention effect in the subgroup of moderately impaired patients, with an MMSE score of $15-20$.

As mentioned, many reasons can explain this difference between proxy-reported and self-reported patient QOL. First, most of the included patients were cared for by their child, which has been reported to be associated with a worse QOL than those cared for by their spouses $[45,50]$. Then, in THERAD, factors that negatively influence proxy reporting, e.g. burden $[52,55]$, were present, with $70 \%$ of caregivers having a moderate or high 
burden ZBI score $>20($ mean $=30.9))$. However, burden is not alleviated by the intervention (it should be mentioned that our intervention was designed to improve caregivers' knowledge, which is known to enhance patient QOL [61], and not to lessen the caregiver's burden, which introduces discrepancies in QOL assessment [52]). We did not specifically provide formal psychological support. Subgroup analyses showed an improvement in caregiver-reported patient QOL among the most exhausted caregivers, particularly at M12, without, surprisingly, any reduced burden, suggesting a potential effect among exhausted caregivers [52].

\section{Limitations}

From a methodological point of view, THERAD has several limitations. The recruitment setting (ambulatory units) induces a selection bias because of a "restraint" profile of $\mathrm{AD}$ patients (severity, autonomy, etc.) and negatively influences the generalizability of the results. However, the randomization secondarily limits this phenomenon. The monocentric design of the study is also a factor limiting generalizability of the results.

Then, as suggested by the improvement in the patients' QOL in both groups, which is not common [50, 53], we probably provided a certain level of unintentional support in the control group despite our effort to limit this bias by designing a real "attention control group". We can imagine our results would have been positive for proxy-rated QOL or more significant for self-rated QOL if we did not pay any attention to the control group (but in this case, it would have been difficult to disentangle attention from the intervention effect). Moreover, even if we tried to offer purely educational information, "informal psychological support" may have occurred during coffee breaks when caregivers shared their caregiving experiences with their counterparts.

Regarding the assessment of the intervention effect, a scale of knowledge [8] or a sense of competence [62] could have been used instead of QOL, despite often previously studied, because embracing all of the dimensions of one's QOL in a formalized, reproducible but tailored format [28] was perhaps too ambitious. However, it seemed reachable in the existing literature at the time we designed the trial. Moreover, some reviews, conducted in other chronic conditions, showed that improving the caregiver's knowledge does not necessarily lead to a change in the way help and care are provided [63].

Last, joint sessions (helpful with regard to communication skills and social abilities) would have been of interest. They will be implemented in our future TPE programme.

However, THERAD presented several strengths.
First, the methodological choice of a randomized controlled trial with an attention control group in such an intervention seems robust. We performed intention-totreat analysis and per-protocol analysis to approach the theoretical effect of our intervention, and we need to emphasize that they produced comparable conclusions. The compliance was good (defined as at least 3 group sessions and the two individual sessions), and in such an educational intervention, especially with a condensed content and short duration, missing one single session was considered as missing a significant "dose" of the intervention.

Then, we used a patient-centred, clinically meaningful, relevant outcome, QOL, rather than an intermediate outcome, such as a process indicator (coping, selfefficacy), thus avoiding previously studied outcomes (burden or caregiver's knowledge and sense of competence [8]).

The patient was fully included in the educational part. We consider our dyadic approach both in the intervention (inclusion of patients in the educational programme) and evaluation (the two perspectives) to be a strength.

As a considerable strength, such approaches will help in the fight against stereotypes [46] and encourage social inclusion.

Additional studies targeting a subpopulation of caregivers (spouse vs. child, male caregivers vs. female caregivers) and also $\mathrm{AD}$ patients (severity of cognitive impairment and BPSD) are necessary using the methodology of an RCT with an attention control group to both limit bias and apprehend the wealth of the two members of the dyad point of view.

\section{Conclusion}

THERAD introduces a double perspective, by proxy and self-reporting, in the assessment of an educational intervention targeting the dyad in $\mathrm{AD}$. Our contrasting results on the patient's QOL underline the challenging issue of measuring the $\mathrm{AD}$ patients' $\mathrm{QOL}$ due to documented bias and the need to find a robust methodological approach. THERAD also suggests the need to design educational interventions targeting subpopulations of caregivers (spouse vs. child, male caregivers vs. female caregivers) and also patients (by the severity of cognitive impairment and BPSD). More generally, attention payed to the AD patient's point of view, introduced into dyadic approaches, is fundamental with social participation and "inclusion" being part of "living well with dementia" [46].

\section{Abbreviations}

AD: Alzheimer's disease; ADL: Activities of daily living; BPSD: Behavioural and psychological symptoms of dementia; CGA: Comprehensive geriatric assessment; GDS: Geriatric Depression Scale; IADL: Instrumental activities of 
daily living; ITT: Intention-to-treat analysis; MMSE: Mini-Mental Score Examination; M0: Baseline visit; M2: 2nd month visit; M6: 6th month visit; M12: 12th month visit; NHP: Nottingham Health Profile; NPI: NeuroPsychiatric Inventory; QOL-AD: Logsdon Quality of Life in Alzheimer's disease; TPE: Therapeutic Patient Education; TUH: Toulouse University Hospital; ZBI: Zarit Burden Interview

\section{Acknowledgements}

The pluriprofessional team of the study: Audrey Zueras, Marie-Pierre Bautrait and Corine Bonnafous in particular. French DGOS of Ministry of Health for Funding. Toulouse University Hospital research and innovation team: MarieElise Llau and Fatiha Boudouh-Ponsard. All participants (patients and caregivers). Anne-Laure Fourrier for help in translation. The Mederic Alzheimer Foundation for research scholarship.

\section{Authors' contributions}

$\mathrm{HV}$ was responsible for the concept of the study, data collection and trial monitoring as the principal investigator, and writer of the manuscript. CC was responsible for data analysis and revision of the manuscript. ED was responsible for acquisition of subjects and collection of data. AP was responsible for acquisition of subjects and collection of data. MS was responsible for revision of the manuscript. VG was responsible for the methodological design of the study, data analysis and revision of the manuscript. All authors have read and approved the final version of the manuscript.

\section{Funding}

This study was funded by the French Ministry of Health (DGOS) (Grant PREQHOS 2011: Afssaps: 2012-A00301-42). The author received a national research scholarship (PhD 2018) from the Mederic Alzheimer Foundation (financial support for communication and dissemination of results once the study was completed)

\section{Availability of data and materials}

The datasets used and/or analysed during the current study are available from the corresponding author on reasonable request.

\section{Declarations}

\section{Ethics approval and consent to participate}

The study protocol was approved by French agencies belonging to the French Ministry of Health (CNIL and ANSM (2012-A00301-42)). The ethics committee of the "Comité de protection des personnes (CPP) South-West of France approved the study.

\section{Consent for publication}

"Not applicable" in this section.

\section{Competing interests}

The authors declare that they have no competing interests in this section.

\section{Author details}

${ }^{1}$ Geriatric Department, Toulouse University Hospital, Hopital La Grave- Cité de la Santé Place Lange TSA 60 033, 31059 Toulouse Cedex 9, France. ${ }^{2}$ Inserm UMR 1295: Center for Research in Population Health (CERPOP) - Department of Epidemiology and Public Health, University of Toulouse, II F-31073, 37, allées Jules Guesde, 31073 Toulouse cedex, France.

Received: 2 June 2021 Accepted: 30 August 2021

Published online: 12 September 2021

\section{References}

1. Korsnes MS, Winkler AS. Global, regional, and national burden of dementia, 1990-2016: Predictions need local calibration. Neurology. 2020;94(16):718-9. https://doi.org/10.1212/WNL.0000000000009301

2. Bouati N, Sagne A, Hunsicker M, Gavazzi G, Couturier P. Exhaustion of family caregivers: a masked domestic crisis? A psychodynamic and systemic approach. Geriatr Psychol Neuropsychiatr Vieil. 2016;14(1):67-76. https://doi. org/10.1684/pnv.2016.0585
3. Cheng S-T. Dementia caregiver burden: a research update and critical analysis. Curr Psychiatry Rep. 2017;19(9):64. https://doi.org/10.1007/s11920017-0818-2.

4. Maher AR, Maglione M, Bagley S, Suttorp M, Hu JH, Ewing B, et al. Efficacy and comparative effectiveness of atypical antipsychotic medications for offlabel uses in adults: a systematic review and meta-analysis. JAMA. 2011; 306(12):1359-69. https://doi.org/10.1001/jama.2011.1360.

5. Brodaty $\mathrm{H}$, Green A, Koschera A. Meta-analysis of psychosocial interventions for caregivers of people with dementia. J Am Geriatr Soc. 2003;51(5):657-64 https://doi.org/10.1034/j.1600-0579.2003.00210.x.

6. Tompkins SA, Bell PA. Examination of a psychoeducational intervention and a respite grant in relieving psychosocial stressors associated with being an Alzheimer's caregiver. J Gerontol Soc Work. 2009;52(2):89-104. https://doi. org/10.1080/01634370802561877

7. Martín-Carrasco M, Martín MF, Valero CP, Millán PR, García Cl, Montalbán SR, et al. Effectiveness of a psychoeducational intervention program in the reduction of caregiver burden in Alzheimer's disease patients' caregivers. Int J Geriatr Psychiatry. 2009;24(5):489-99. https://doi.org/10.1002/gps.2142.

8. Tang S-H, Chio O-I, Chang L-H, Mao HF, Chen LH, Yip PK, et al. Caregiver active participation in psychoeducational intervention improved caregiving skills and competency. Geriatr Gerontol Int. 2018;18(5):750-7. https://doi. org/10.1111/ggi.13246.

9. Smits CHM, de Lange J, Dröes R-M, Meiland F, Vernooij-Dassen M, Pot AM. Effects of combined intervention programmes for people with dementia living at home and their caregivers: a systematic review. Int J Geriatr Psychiatry. 2007;22(12):1181-93. https://doi.org/10.1002/gps.1805.

10. Livingston G, Manela M, O'Keeffe A, et al. Clinical effectiveness of the START (STrAtegies for RelaTives) psychological intervention for family carers and the effects on the cost of care for people with dementia: 6-year follow-up of a randomised controlled trial. Br J Psychiatry J Ment Sci. 2020;216(1):3542. https://doi.org/10.1192/bjp.2019.160

11. Lee M, Ryoo JH, Crowder J, Byon HD, Williams IC. A systematic review and meta-analysis on effective interventions for health-related quality of life among caregivers of people with dementia. J Adv Nurs. 2020;76(2):475-89. https://doi.org/10.1111/jan.14262.

12. Devor M, Renvall M. An educational intervention to support caregivers of elders with dementia. Am J Alzheimers Dis Other Demen. 2008;23(3):23341. https://doi.org/10.1177/1533317508315336.

13. Parker D, Mills S, Abbey J. Effectiveness of interventions that assist caregivers to support people with dementia living in the community: a systematic review. Int J Evid Based Healthc. 2008;6(2):137-72. https://doi.org/10.1111/j.1 744-1609.2008.00090.x

14. Bessey LJ, Walaszek A. Management of behavioral and psychological symptoms of dementia. Curr Psychiatry Rep. 2019;21(8):66. https://doi.org/1 0.1007/s11920-019-1049-5.

15. Vandepitte S, Van Den Noortgate N, Putman K, Verhaeghe S, Faes K, Annemans L. Effectiveness of supporting informal caregivers of people with dementia: a systematic review of randomized and non-randomized controlled trials. J Alzheimers Dis JAD. 2016;52(3):929-65. https://doi.org/1 0.3233/JAD-151011.

16. Mittelman MS, Ferris SH, Shulman E, Steinberg G, Levin B. A family intervention to delay nursing home placement of patients with Alzheimer disease. A randomized controlled trial. JAMA. 1996;276(21):1725-31. https:// doi.org/10.1001/jama.1996.03540210033030.

17. Bruvik FK, Allore HG, Ranhoff AH, Engedal K. The effect of psychosocial support intervention on depression in patients with dementia and their family caregivers: an assessor-blinded randomized controlled trial. Dement Geriatr Cogn Disord Extra. 2013;3(1):386-97. https://doi.org/10.1159/0003 55912.

18. de Rotrou J, Cantegreil I, Faucounau V, Wenisch E, Chausson C, Jegou D, et al. Do patients diagnosed with Alzheimer's disease benefit from a psychoeducational programme for family caregivers? A randomised controlled study. Int J Geriatr Psychiatry. 2011;26(8):833-42. https://doi.org/10.1002/gps.2611.

19. Waldorff FB, Buss DV, Eckermann A, et al. Efficacy of psychosocial intervention in patients with mild Alzheimer's disease: the multicentre, rater blinded, randomised Danish Alzheimer Intervention Study (DAISY). BMJ. 2012;345(jul17 1):e4693. https://doi.org/10.1136/bmj.e4693.

20. Margat A, Gagnayre R, Lombrail P, de Andrade V, Azogui-Levy S. Health literacy and patient education interventions: a review. Sante Publique Vandoeuvre--Nancy Fr. 2017;29(6):811-20. https://doi.org/10.3917/spub.176. 0811. 
21. Oh ES, Rabins PV. Dementia. Ann Intern Med. 2019;171(5):ITC33-48. https:// doi.org/10.7326/AlTC201909030.

22. Gibson PG, Powell H, Coughlan J, et al. Self-management education and regular practitioner review for adults with asthma. Cochrane Database Syst Rev. 2003;1:CD001117. https://doi.org/10.1002/14651858.CD001117.

23. Rueda S, Park-Wyllie LY, Bayoumi AM, Tynan AM, Antoniou T, Rourke S, et al. Patient support and education for promoting adherence to highly active antiretroviral therapy for HIV/AIDS. Cochrane Database Syst Rev. 2006;3: CD001442. https://doi.org/10.1002/14651858.CD001442.pub2.

24. Meng K, Seekatz B, Haug G, Mosler G, Schwaab B, Worringen U, et al. Evaluation of a standardized patient education program for inpatient cardiac rehabilitation: impact on illness knowledge and self-management behaviors up to 1 year. Health Educ Res. 2014;29(2):235-46. https://doi.org/1 0.1093/her/cyt107.

25. Piersol CV, Canton K, Connor SE, Giller I, Lipman S, Sager S. Effectiveness of interventions for caregivers of people with Alzheimer's disease and related major neurocognitive disorders: a systematic review. Am J Occup Ther Off Publ Am Occup Ther Assoc. 2017;71(5):7105180020p1-7105180020p10. https://doi.org/10.5014/ajot.2017.027581

26. Williams KW, Word C, Streck MR, Titus MO. Parental education on asthma severity in the emergency department and primary care follow-up rates. Clin Pediatr (Phila). 2013;52(7):612-9. https://doi.org/10.1177/00099228134 79163.

27. Shimodera S, Furukawa TA, Mino Y, Shimazu K, Nishida A, Inoue S. Costeffectiveness of family psychoeducation to prevent relapse in major depression: results from a randomized controlled trial. BMC Psychiatry. 2012; 12(1):40. https://doi.org/10.1186/1471-244X-12-40.

28. Moon $\mathrm{H}$, Adams KB. The effectiveness of dyadic interventions for people with dementia and their caregivers. Dement Lond Engl. 2013;12(6):821-39. https://doi.org/10.1177/1471301212447026.

29. Villars H, Gardette V, Perrin A, Hein C, Elmalem S, de Peretti E, et al. Study protocol: randomised controlled trial to evaluate the impact of an educational programme on Alzheimer's disease patients' quality of life. Alzheimers Res Ther. 2014;6(5-8):66. https://doi.org/10.1186/s13195-0140066-1.

30. Folstein MF, Folstein SE, McHugh PR. "Mini-mental state". A practical method for grading the cognitive state of patients for the clinician. J Psychiatr Res. 1975;12(3): 189-98. https:/doi.org/10.1016/0022-3956(75)90026-6.

31. Nourhashemi F, Andrieu S, Gillette-Guyonnet S, Giraudeau B, Cantet C, Coley $\mathrm{N}$, et al. Effectiveness of a specific care plan in patients with Alzheimer's disease: cluster randomised trial (PLASA study). BMJ. 2010; 340(jun03 1):c2466. https://doi.org/10.1136/bmj.c2466.

32. Teri L, McCurry SM, Logsdon R, Gibbons LE. Training community consultants to help family members improve dementia care: a randomized controlled trial. Gerontologist. 2005;45(6):802-11. https://doi.org/10.1093/geront/45.6. 802.

33. Villars H, Dupuy C, Perrin A, Vellas B, Nourhashemi F. Impact of a therapeutic educational program on quality of life in Alzheimer's disease: results of a pilot study. J Alzheimers Dis JAD. 2015;43(1):167-76. https://doi. org/10.3233/JAD-141179.

34. Bruttomesso D, Gagnayre R, Leclercq D, Crazzolara D, Busata E, d'Ivernois JF, et al. The use of degrees of certainty to evaluate knowledge. Patient Educ Couns. 2003;51(1):29-37. https://doi.org/10.1016/s0738-3991(02)00226-4.

35. Logsdon RG, Gibbons LE, McCurry SM, Teri L. Assessing quality of life in older adults with cognitive impairment. Psychosom Med. 2002;64(3):510-9. https://doi.org/10.1097/00006842-200205000-00016.

36. Cummings JL, Mega M, Gray K, Rosenberg-Thompson S, Carusi DA, Gornbein J. The Neuropsychiatric Inventory: comprehensive assessment of psychopathology in dementia. Neurology. 1994;44(12):2308-14. https://doi. org/10.1212/wnl.44.12.2308

37. Katz S, Downs TD, Cash HR, Grotz RC. Progress in development of the index of ADL. Gerontologist. 1970;10(1):20-30. https://doi.org/10.1093/geront/1 0.1 part_1.20.

38. Lawton MP, Brody EM. Assessment of older people: self-maintaining and instrumental activities of daily living. Gerontologist. 1969;9(3):179-86 https://doi.org/10.1093/geront/9.3_Part_1.179.

39. Zarit SH, Reever KE, Bach-Peterson J. Relatives of the impaired elderly: correlates of feelings of burden. Gerontologist. 1980;20(6):649-55. https:// doi.org/10.1093/geront/20.6.649.

40. Boyer F, Novella J-L, Morrone I, Jolly D, Blanchard F. Agreement between dementia patient report and proxy reports using the Nottingham Health
Profile. Int J Geriatr Psychiatry. 2004;19(11):1026-34. https://doi.org/10.1002/ gps.1191.

41. Barbe C, Jolly D, Morrone I, Wolak-Thierry A, Dramé M, Novella JL, et al. Factors associated with quality of life in patients with Alzheimer's disease. BMC Geriatr. 2018;18(1):159. https://doi.org/10.1186/s12877-018-0855-7.

42. Clément JP, Nassif RF, Léger JM, Marchan F. Development and contribution to the validation of a brief French version of the Yesavage Geriatric Depression Scale. L'Encephale. 1997;23(2):91-9.

43. Twisk J, Bosman L, Hoekstra T, Rijnhart J, Welten M, Heymans M. Different ways to estimate treatment effects in randomised controlled trials. Contemporary Clinical Trials Communications. 2018;10:80-5.

44. Trigg R, Jones RW, Knapp M, King D, Lacey LA. DADE-2 Investigator Groups. The relationship between changes in quality of life outcomes and progression of Alzheimer's disease: results from the dependence in $A D$ in England 2 longitudinal study. Int J Geriatr Psychiatry. 2015;30(4):400-8. https://doi.org/10.1002/gps.4150.

45. Schumann C, Alexopoulos P, Perneczky R. Determinants of self- and carerrated quality of life and caregiver burden in Alzheimer disease. Int I Geriatr Psychiatry. 2019;34(10):1378-85. https://doi.org/10.1002/gps.5126.

46. Morgan J. Living well with dementia. Lancet Neurol. 2018;17(4):306-7. https://doi.org/10.1016/S1474-4422(17)30424-6.

47. Schölzel-Dorenbos CJM, Ettema TP, Bos J, Boelens-van der Knoop E, Gerritsen DL, Hoogeveen F, et al. Evaluating the outcome of interventions on quality of life in dementia: selection of the appropriate scale. Int J Geriatr Psychiatry. 2007;22(6):511-9. https://doi.org/10.1002/gps.1719.

48. Hurt CS, Banerjee S, Tunnard C, Whitehead D, Tsolaki M, Mecocci P, et al. Insight, cognition and quality of life in Alzheimer's disease. J Neurol Neurosurg Psychiatry. 2010;81(3):331-6. https://doi.org/10.1136/jnnp.2009.1 84598.

49. Rabins PV, Black BS. Measuring quality of life in dementia: purposes, goals, challenges and progress. Int Psychogeriatr. 2007;19(3):401-7. https://doi. org/10.1017/S1041610207004863.

50. Conde-Sala JL, Garre-Olmo J, Turró-Garriga O, López-Pousa S, Vilalta-Franch J. Factors related to perceived quality of life in patients with Alzheimer's disease: the patient's perception compared with that of caregivers. Int J Geriatr Psychiatry. 2009;24(6):585-94. https://doi.org/10.1002/gps.2161.

51. Missotten P, Dupuis G, Adam S. Dementia-specific quality of life instruments: a conceptual analysis. Int Psychogeriatr. 2016;28(8):1245-62. https://doi.org/10.1017/S1041610216000417.

52. Andrieu S, Coley N, Rolland Y, Cantet C, Arnaud C, Guyonnet S, et al. Assessing Alzheimer's disease patients' quality of life: discrepancies between patient and caregiver perspectives. Alzheimers Dement J Alzheimers Assoc. 2016;12(4):427-37. https://doi.org/10.1016/j.jalz.2015.09.003.

53. Bosboom PR, Alfonso H, Almeida OP. Determining the predictors of change in quality of life self-ratings and carer-ratings for community-dwelling people with Alzheimer disease. Alzheimer Dis Assoc Disord. 2013;27(4):36371. https://doi.org/10.1097/WAD.0b013e318293b5f8.

54. Sands LP, Ferreira P, Stewart AL, Brod M, Yaffe K. What explains differences between dementia patients' and their caregivers' ratings of patients' quality of life? Am J Geriatr Psychiatry Off J Am Assoc Geriatr Psychiatry. 2004;12(3): 272-80. https://doi.org/10.1097/00019442-200405000-00006.

55. Zucchella C, Bartolo M, Bernini S, Picascia M, Sinforiani E. Quality of life in Alzheimer disease: a comparison of patients' and caregivers' points of view. Alzheimer Dis Assoc Disord. 2015;29(1):50-4. https://doi.org/10.1097/WAD. 0000000000000050.

56. Sousa MFB, Santos RL, Arcoverde C, Simões P, Belfort T, Adler I, et al. Quality of life in dementia: the role of non-cognitive factors in the ratings of people with dementia and family caregivers. Int Psychogeriatr. 2013;25(7):1097-105. https://doi.org/10.1017/S1041610213000410.

57. Zhao H, Novella J-L, Dramé M, Mahmoudi R, Barbe C, di Pollina L, et al. Factors associated with caregivers' underestimation of quality of life in patients with Alzheimer's disease. Dement Geriatr Cogn Disord. 2012;33(1): 11-7. https://doi.org/10.1159/000333070.

58. Jing W, Willis R, Feng Z. Factors influencing quality of life of elderly people with dementia and care implications: a systematic review. Arch Gerontol Geriatr. 2016;66:23-41. https://doi.org/10.1016/j.archger.2016.04.009.

59. Thorgrimsen L, Selwood A, Spector A, Royan L, de Madariaga Lopez M, Woods RT, et al. Whose quality of life is it anyway? The validity and reliability of the Quality of Life-Alzheimer's Disease (QoL-AD) scale. Alzheimer Dis Assoc Disord. 2003;17(4):201-8. https://doi.org/10.1097/00002 093-200310000-00002. 
60. Chappell NL, Dujela C, Smith A. Spouse and adult child differences in caregiving burden. Can J Aging Rev Can Vieil. 2014;33(4):462-72. https://doi. org/10.1017/S0714980814000336.

61. Ying J, Wang Y, Zhang M, Wang S, Shi Y, Li H, et al. Effect of

multicomponent interventions on competence of family caregivers of people with dementia: a systematic review. J Clin Nurs. 2018;27(9-10):174458. https://doi.org/10.1111/jocn.14326.

62. Vernooij-Dassen M, Kurz X, Scuvee-Moreau J, Dresse A. The measurement of sense of competence in caregivers of patients with dementia. Rev Epidemiol Sante Publique. 2003;51(2):227-35.

63. Li T, Wu HM, Wang F, Huang CQ, Yang M, Dong BR, et al. Education programmes for people with diabetic kidney disease. Cochrane Database Syst Rev. 2011;6:CD007374. https://doi.org/10.1002/14651858.CD007374. pub2.

\section{Publisher's Note}

Springer Nature remains neutral with regard to jurisdictional claims in published maps and institutional affiliations.

Ready to submit your research? Choose BMC and benefit from:

- fast, convenient online submission

- thorough peer review by experienced researchers in your field

- rapid publication on acceptance

- support for research data, including large and complex data types

- gold Open Access which fosters wider collaboration and increased citations

- maximum visibility for your research: over $100 \mathrm{M}$ website views per year

At BMC, research is always in progress.

Learn more biomedcentral.com/submissions 